University of Louisville

ThinkIR: The University of Louisville's Institutional Repository

Electronic Theses and Dissertations

8-2010

\title{
Numerical investigation of the aerodynamic and structural characteristics of a corrugated wing.
}

Kyle Hord

University of Louisville

Follow this and additional works at: https://ir.library.louisville.edu/etd

\section{Recommended Citation}

Hord, Kyle, "Numerical investigation of the aerodynamic and structural characteristics of a corrugated wing." (2010). Electronic Theses and Dissertations. Paper 634.

https://doi.org/10.18297/etd/634

This Master's Thesis is brought to you for free and open access by ThinkIR: The University of Louisville's Institutional Repository. It has been accepted for inclusion in Electronic Theses and Dissertations by an authorized administrator of ThinkIR: The University of Louisville's Institutional Repository. This title appears here courtesy of the author, who has retained all other copyrights. For more information, please contact thinkir@louisville.edu. 
NUMERICAL INVESTIGATION OF THE AERODYNAMIC AND STRUCTURAL CHARACTERISTICS OF A CORRUGATED WING

\author{
By \\ Kyle Hord \\ B.S. Eng., University of Louisville, 2009
}

\begin{abstract}
A Thesis
Submitted to the Faculty of the University of Louisville J.B. Speed School of Engineering In Partial Fulfillment of Requirements For the Professional Degree
\end{abstract}

\title{
MASTER OF ENGINEERING
}

Department of Mechanical Engineering

August 2010 

NUMERICAL INVESTIGATION OF THE AERODYNAMIC AND STRUCTURAL CHARACTERISTICS OF A CORRUGATED WING

Submitted by:

Kyle Hord

A Thesis Approved on

(Date)

by the Following Reading and Examination Committee:

Dr. Yongsheng Lian, Thesis Director

Mechanical Engineering

Dr. Michael Ol

Wright-Patterson Air Force Base

Dr. Glen Prater

Mechanical Engineering

Dr. Rammohan Ragade

Computer Engineering and Computer Sciences 


\section{ACKNOWLEDGEMENTS}

This work is partially support by a grant from the Kentucky Science and Engineering Foundation and by an Intramural Research Incentive Grant from the Office of the Executive Vice President for Research at the University of Louisville.

Special thanks to Dr. Yongsheng Lian for his support and guidance. Without him, this project would have never come to into existence. He provided the tools and funding necessary to perform this study. Also for never giving up on me, for I am sure, I irritated him quite a few times. If Dr. Lian never gave me a chance when I first started studying under him, I would have never realized my interested in computational fluid dynamics. 


\begin{abstract}
Previous experimental studies on static, bio-inspired corrugated wings have shown that they produce favorable aerodynamic properties such as delayed stall compared to streamlined wings and flat plates at high Reynolds numbers $\left(\operatorname{Re} \geq 4 \times 10^{4}\right)$. The majority of studies have been carried out with scaled models of dragonfly forewings from the Aeshna Cyanea in either wind tunnels or water channels. In this thesis, the aerodynamics of a corrugated airfoil was studied using computational fluid dynamics methods at a low Reynolds number of 1000. Structural analysis was also performed using the commercial software SolidWorks 2009. The flow field is described by solving the incompressible Navier-Stokes equations on an overlapping grid using the pressure-Poisson method. The equations are discretized in space with second-order accurate central differences. Time integration is achieved through the second-order Crank-Nicolson implicit method. The complex vortex structures that form in the corrugated airfoil valleys and around the corrugated airfoil are studied in detail. Comparisons are made with experimental measurements from corrugated wings and also with simulations of a flat plate. Contrary to the studies at high Reynolds numbers, our study shows that at low Reynolds numbers the wing corrugation does not provide any aerodynamic benefit compared to a smoothed flat plate. Instead, the corrugated profile generates more pressure drag which is only partially offset by the reduction of friction drag, leading to more total drag than the flat plate. Structural analysis shows
\end{abstract}


that the wing corrugation can increase the resistance to bending moments on the wing structure. A smoothed structure has to be three times thicker to provide the same stiffness. It was concluded the corrugated wing has the structural benefit to provide the same resistance to bending moments with a much reduced weight. 
NOMENCLATURE

$\begin{array}{llll}\alpha & = & \text { Angle of attack } \\ c & = & \text { Chord } \\ c_{D}= & \text { Coefficient of drag } \\ c_{L}= & \text { Coefficient of lift } \\ D= & \text { Drag } \\ f & = & \text { Wake vortex shedding frequency } \\ L & = & \text { Lift } \\ p & = & \text { Pressure } \\ \operatorname{Pr}= & \text { Prandtl number } \\ R e= & \text { Reynold's number, } \rho \mathrm{U}_{\infty} \mathrm{c} / \mu \\ S t= & \text { Strouhal number, } f h / \mathrm{U}_{\infty} \\ h & = & \text { Characteristic height of the object, } \cos (\alpha) * \mathrm{c} \\ t & = & \text { Time } \\ \mathrm{t}_{\mathrm{a}}= & \text { Airfoil thickness } \\ \mathrm{T} & = & \text { Temperature } \\ \vec{u} & = & \text { Velocity field }(u, v, w) \\ u & = & \text { X-component velocity } \\ U_{\infty}= & \text { Free stream velocity } \\ \tau & = & \text { Viscous stress } \\ \mu & = & \text { Viscosity } \\ \rho & = & \text { Density }\end{array}$




\section{TABLE OF CONTENTS}

\section{Page}

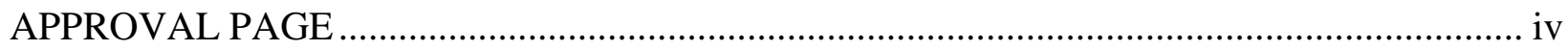

ACKNOWLEDGEMENTS ....................................................................................... ii

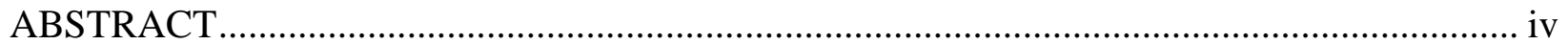

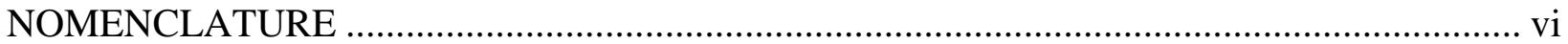

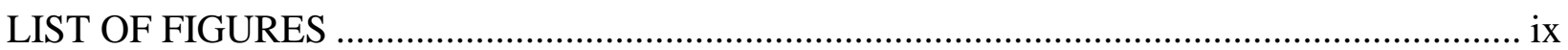

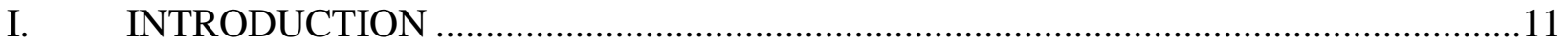

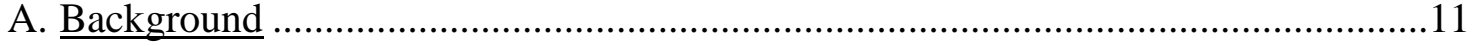

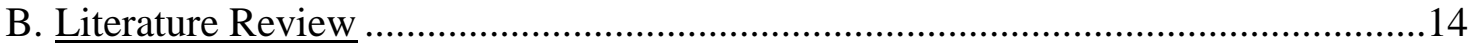

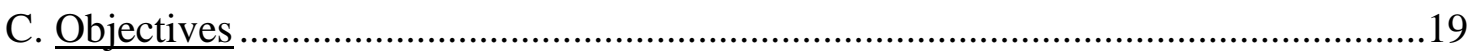

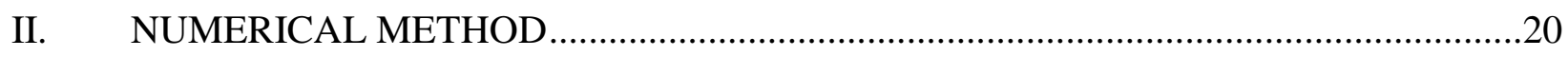

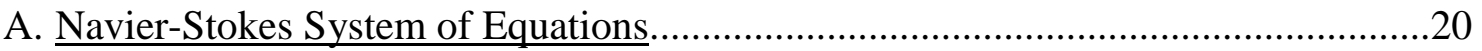

B. Solving the Incompressible Navier Stokes Equation ................................................25

C. Boundary Conditions .....................................................................................

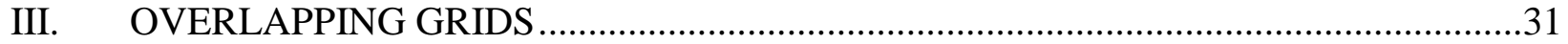

A. Types of Grid Generation .................................................................................

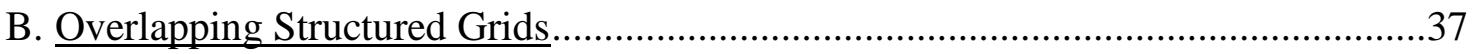

C. Overlapping Grid Assembly................................................................................

D. Geometry Selection ..........................................................................................

E. Corrugated Profile Grid Generation ……....................................................................46

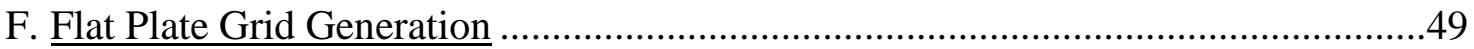

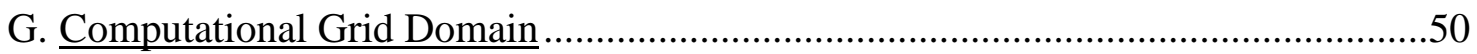

IV. CODE VALIDATION AND COMPUTATIONAL SETUP ………............................51

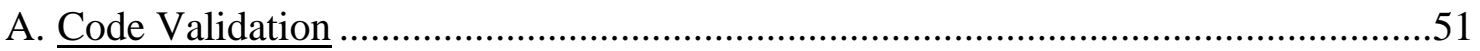

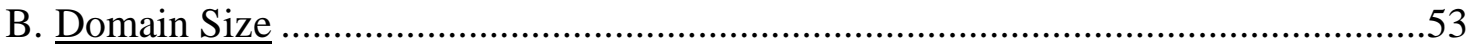

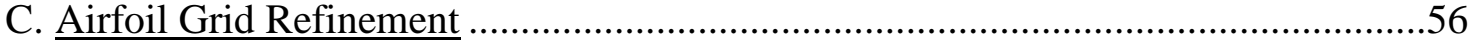




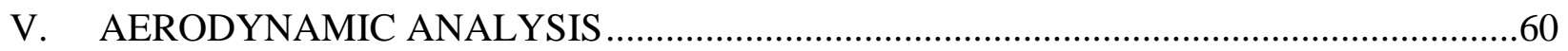

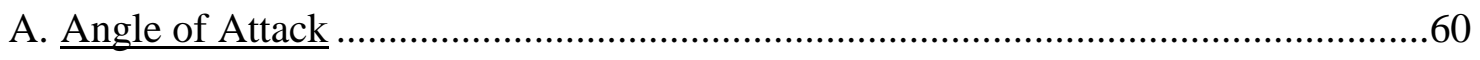

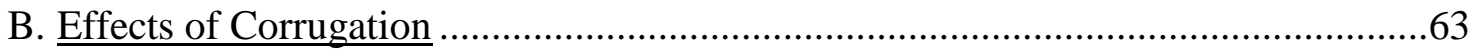

C. Stability of Corrugated Profile …………..................................................................65

D. Viability of Corrugated Profile ..........................................................................67

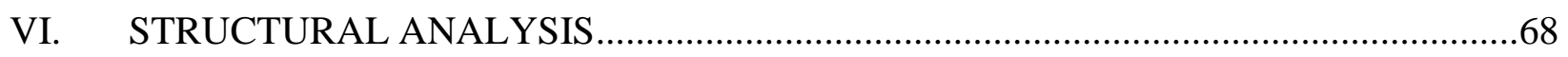

A. Material Properties and Modeling ...........................................................................68

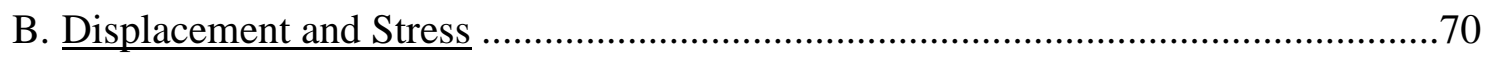

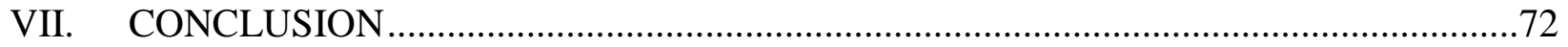

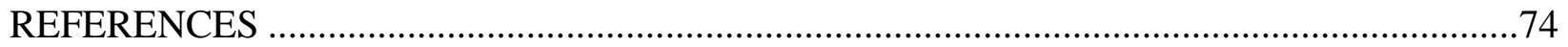

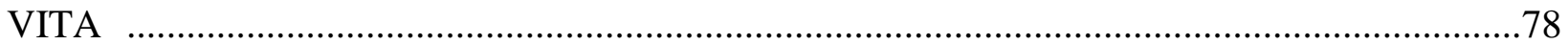




\section{LIST OF FIGURES}

$\underline{\text { Page }}$

FIGURE 1 - Gliding ratio as a function of chord Reynolds number (McMasters and Henderson 1980) .12

FIGURE 2 - Aeshan Cyana (Southern Hawker Dragonfly). .15

FIGURE 3 - Cross section of an Aeshna Cyanea's forewing at mid-span (Murphy et al. 2009).15

FIGURE 4 - Process diagram of split-step scheme of the Navier-Stokes equation..... .28

FIGURE 5 - A structured grid meshed between an annulus and square. .32

FIGURE 6 - A multiblocked structured grid. 33

FIGURE 7 - Unstructured annulus and square mesh. .34

FIGURE 8 - Cartesian grid of annulus and square. .35

FIGURE 9 - Example of Overlapping Structured Grid system (Guerrero 2009, Henshaw 1998) 37 FIGURE 10 - Component grids a Cartesian grid, G1, and a body-fitted structured grid around an annulus, G2. (Guerrero 2009, Henshaw 1998) 40

FIGURE 11 - Identifying unused, and interpolation points (Henshaw 1998, Guerrero 2009)......41

FIGURE 12 - Completed Overlapping grid system (Guerrero 2009, Henshaw 1998)................42

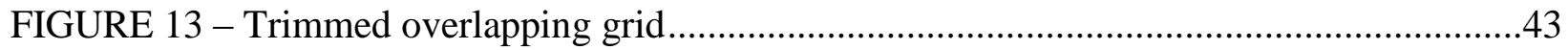

FIGURE 14 - Aeshan Cyana cross section profiles .............................................................44

FIGURE 15 - Corrugated profile created by Murphy (2009). .............................................45

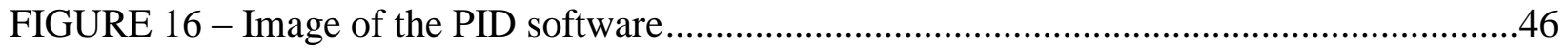

FIGURE 17 - Spline mapping of the corrugated profile ..................................................46

FIGURE 18 - Structured grids grown in the normal direction of surface ................................47

FIGURE 19 - Hyperbolic grid generation no user control ..................................................48

FIGURE 20 - Hyperbolic grid generation with user control ..............................................48

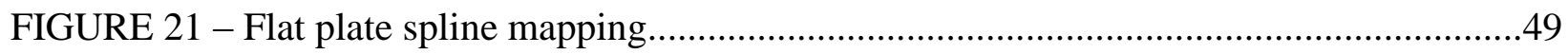

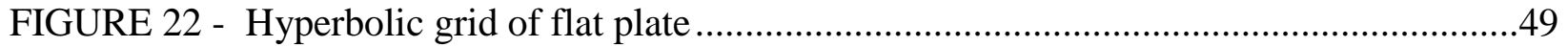

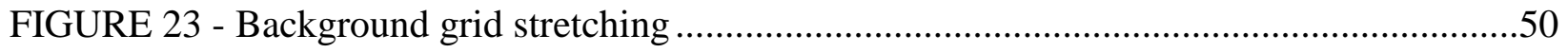


FIGURE 24 - Overlapping grid layout used for code validation ....

FIGURE 25 - Schematic of computational domain.

FIGURE 26 - Effect of domain size on coefficient of lift..................................................55

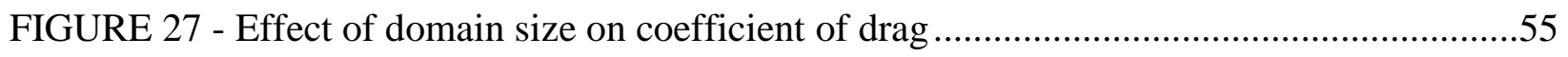

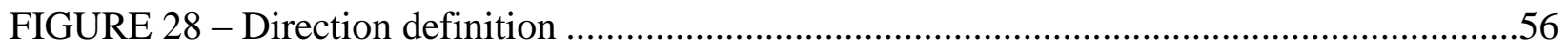

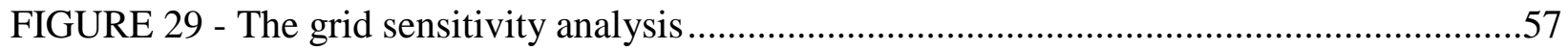

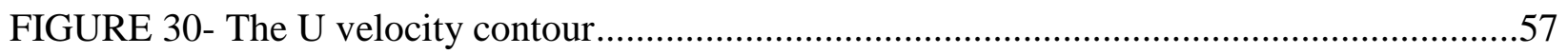

FIGURE 31 - The grid sensitivity with trailing grid........................................................58

FIGURE 32 - Numerical results of corrugated profile plotted against published data................61

FIGURE 33 - Results plotted against numerical results of the flat plate.................................61

FIGURE 34 - Steady flow streamline visualization around the corrugated profile. Virtual profile

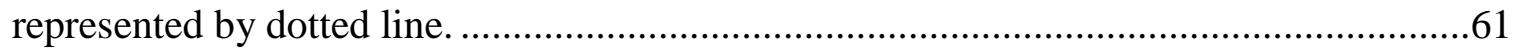

FIGURE 34 - Steady flow streamline visualization around the corrugated profile. Virtual profile

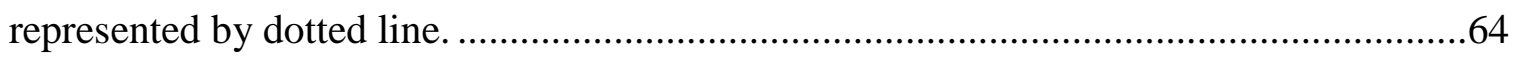

FIGURE 35 - (a) Strouhal number of wake shedding frequency. (b) Oscillating coefficient of lifts . .66

FIGURE 36 - Force history of Flat Plate and Corrugated Airfoil at $20^{\circ}$ .66

FIGURE 37 - Vorticity contours of the corrugated airfoil (left column) and flat plate (right column) at $20^{\circ}$ after periodic flow has been established. .66

FIGURE 38 - The flat plate and corrugated wing models used 69

FIGURE 39 - (a) Max displacement of models (b) Average Von Mises Stress .70 


\section{INTRODUCTION}

\section{A. Background}

Micro-Air-Vehicles (MAVs) are small aerial vehicles with a typical wing span of less than $15 \mathrm{~cm}$ and weigh of less than 90 grams. Their flight speed usually ranges from $2 \mathrm{~m} / \mathrm{s}$ to 10 $\mathrm{m} / \mathrm{s}$. The development of MAVs is of great interest to both military and civilian applications. MAVs can be used in various scenarios such as reconnaissance, surveillance, targeting, search and rescue, and biochemical sensing in confined or otherwise hazardous conditions. Their applications are similar to their larger cousin, the unmanned aerial vehicle (UAV), but MAVs have much smaller sizes and much lower cost. As an example, the General Atomics Aeronautical Systems' Predator ${ }^{1}$ UAV has a cruise speed about $35 \mathrm{~m} / \mathrm{s}$, a wing span of $14.8 \mathrm{~m}$, and cost upwards of approximately $\$ 10$ million. The goal of MAV design is to fit the capabilities of a UAV into a far smaller aircraft.

\footnotetext{
${ }^{1}$ http://www.ga-asi.com/
} 
While there is large potential for the use of MAVs, there are several problems that must be overcome due to their small size and low flight speed. The low Reynolds number presents new aerodynamic challenges in efficient lift generation.

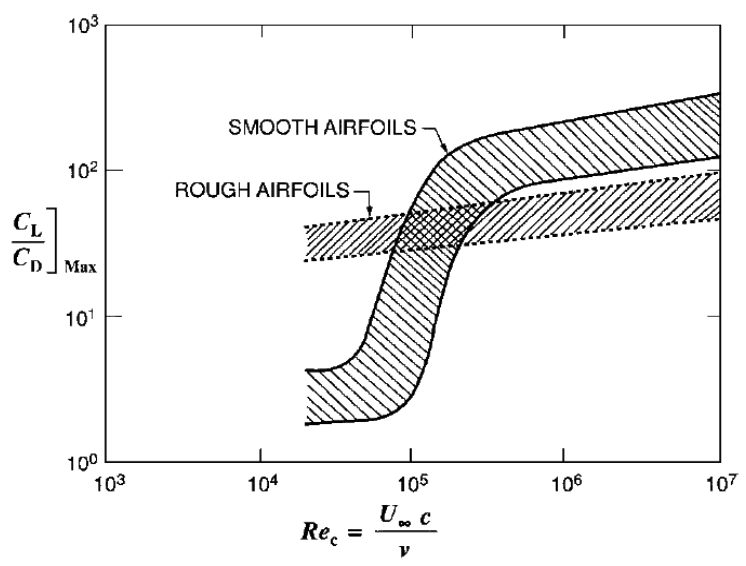

FIGURE 1 - Gliding ratio as a function of chord Reynolds number (McMasters and Henderson 1980)

Due to MAV's slow flight speed, the chord Reynolds number across the wing is low, $10^{2}-10^{4}$. At such a low Reynolds number, the flow across the wing is typically laminar. Current MAV designs rely on conventional streamlined airfoils for lift generation. However, conventional airfoils are designed to operate at much higher Reynolds numbers greater than $10^{5}$. The performance of smooth airfoils deteriorates once the Reynolds number drops below $10^{5}$. Figure 1 is a reproduction of a plot from McMasters and Henderson (1990) maximum lift-to-drag ratios of airfoils as a function of Reynolds number. The lift-to-drag ratio is a measurement of the effectiveness of an airfoil which is proportional to the gliding ratio and climbing ability of the airfoil (Gad-el-Hak 2001). For large aircraft the boundary layers usually transitions to turbulent flow before separation. The turbulent flow can stay attached through more severe adverse pressure gradients. As the Reynolds number falls below $10^{5}$, flow approaches the adverse pressure region as laminar flow. Since laminar flow cannot resist strong adverse pressure 
gradient, flow separates as laminar flow. Laminar flow separation typically leads to premature stall, q dramatic decrease in the lift, and increase in the drag (Gad-el-Hak 2001). To mediate the effects of stalling conditions and the resulting poor lift-to-drag ratio in the low Reynolds number region, new airfoil designs must be created. 


\section{B. Literature Review}

Dragonflies are known for their exceptional flying performance and their gliding ability. Dragonflies are highly maneuverable; they can fly forward and backward, and hover for a long period of time. These characteristics are very desirable for MAV designs. The dragonfly genus Aeschna is capable of gliding for up to 30 seconds without an appreciable loss in altitude (Brodsky 1994). Gliding flight, which requires no energy expenditure, would prove to be a major advantage to MAVs as a power saving technique (Vargas et al. 2004). Their superior gliding performance is due to their high wing aspect ratios. It is well known that wings with high aspect ratios perform exemplarly in gliding flight. Dragonflies have some of the highest aspect ratio wings in the insect world, which naturally give them superior gliding performances compared to other insects. Aeshna juncea (Hawker dragonfly) was calculated to have an aspect ratio of 11.63 and 8.4 for the forewing and hindwing respectively. Other high aspect ratio insects are the Tipula Paludosa (Crane fly) with an aspect ratio of 11.3, the Episyrphus balteatus (Marmalade fly) of 8.45, the Eristalis tenax (Drone fly) of 7.15, and the Bombus lucorum (White-tailed Bumble bee) of 6.83 (Brodsky1994, Vargas et al. 2004)

Besides their high wing aspect ratios, another characteristic that interests researchers is their corrugated structure. Examining an Aeshna Cyanea's forewing reveals a highly corrugated structure where the corrugation varies spanwise and chordwise along the wing. Figure 3 shows a cross section of the wing. The corrugation provides stiffening of the wing while allowing torsion to occur. Because of the dragonfly's unusual wing structure, it does not resemble traditional airfoils; it was originally assumed that such a wing performs poorly in flight with low lift and 
high drag due to this unusual structure. However several studies have led to multiple conclusions about the use of the corrugated structure.

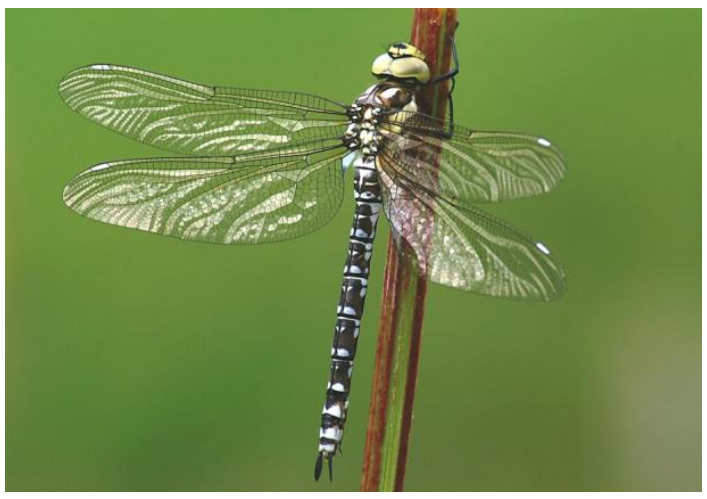

FIGURE 2 - Aeshan Cyana (Southern Hawker Dragonfly)

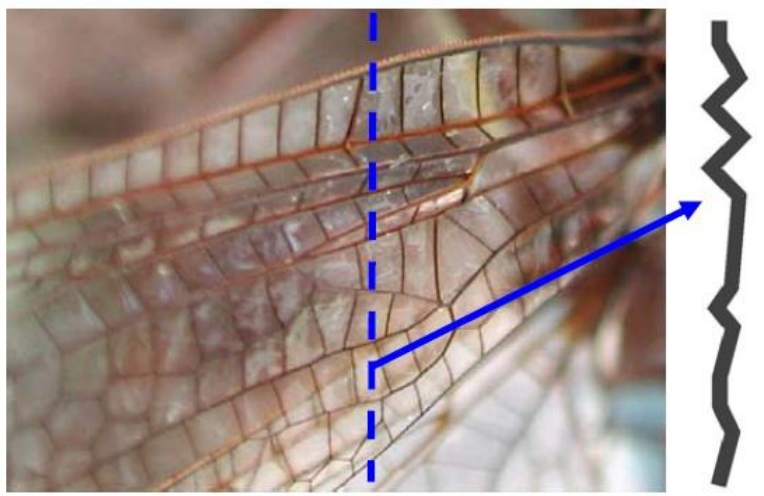

FIGURE 3 - Cross section of an Aeshna Cyanea's forewing at mid-span (Murphy et al. 2009)

Two early wind tunnel experiments conducted on corrugated wings by Rees (1975) and Rudolph (1977) concluded that the corrugation provides no aerodynamic benefits. However they both suggested that fluid flowing over the corrugation could become trapped inside the folds. This trapped fluid would either rotate slowly or remain stagnant. Rudolph also noted that the only benefit a corrugated wing would have over a smooth airfoil is that the corrugation would delay flow separation at higher angles of attack. 
Kesel (1997) extracted three profiles from the forewing of an Aeshna Cyanea. Each profile was taken from different wing span distances. The aerodynamic performance was measured at a chord Reynolds number of 7880 or 10,000, depending on the profile used. Kesel noticed vortices that formed in the wing's corrugation effectively changed the wing shape and made the corrugated wings behave more like a streamlined wing. It was found the profiles behaved similarly to asymmetrical smooth profiles or a flat plate. Kesel concluded that an increase in lift generation cannot be obtained from the vortex system arising from random or uniform corrugations, but only from corrugations finely tuned through evolution.

Murphy and Hu (2009) studied the aerodynamic characteristics of a corrugated airfoil, taken from an Aeshna Cyanea, and compared it with a streamlined airfoil and a flat plate at chord Reynolds numbers of 58,000 and 125,000. The aerodynamic forces measured showed that the corrugated airfoil could generate higher lift, and delay flow separation at higher angles of attacked compared to the streamlined airfoil and flat plate. Using a wind tunnel equipped with a Particle Image Velocimetry, they were able to capture images of vortex structures that form inside the valleys of the corrugation. It was determined that the peaks of the corrugation promoted the flow transition from laminar to turbulent flow, and unsteady vortexes would be captured in the valleys. These vortexes would pull the boundary layer to the surface, keeping the flow attached. This effect allows the turbulent flow to stay attached through more severe pressure gradients.

Vargas et al. (2004) numerically studied a corrugated wing and further compared to a streamlined profiled version of the corrugated wing and a flat plate at Reynolds numbers of 500 through 10,000. The flat plate was found to perform best at low Reynolds numbers of less than 
5,000. Increasing the Reynolds number to 10,000 , it was found the corrugated wing performed better. Vargas et al. concluded that the corrugated wing preformed best at low angles of attack. At these angles, the wing experienced increases in pressure drag, but had a reduction in viscous drag due to the existence of recirculation zones inside the corrugations, leading to a negative shear stress contribution.

Recently Kim et al. (2009) preformed a numerical study on the corrugated wing but with Reynolds numbers of 150, 1400, and 10,000 and angles of attach varying from $0^{\circ}-40^{\circ}$. The study primarily focused on the effects of the corrugation on lift. Kim et al. concluded that the lift was not directly influenced by the Reynolds number, and that the corrugation increased the lift at all the angles tested.

Sunada et al. (2002) performed water tunnel tests to study the characteristics of 20 wings with identical ratio of 7.25 . They found that a $5 \%$ camber wing with sharp leading edge produces better hydrodynamic performance. They also noticed that wing corrugation could also improve the aerodynamic performance. Corrugation was found to be effective because it could increase rigidity. The corrugation makes the thinner wing stronger against bending and torsional moments so the wing can maintain its profile.

Kesel et al. (1998) also demonstrated the structure benefits of a corrugated wing through a finite element analysis. They found that the wing was stabilized primarily by 3-dimensional corrugations. These corrugations dramatically reduced wing defection by a factor of 20 and reduced stress by $95 \%$ compared to a flatter 2-dimensional wing. Kesel concluded that a corrugated profile yielded greater structural stability using less material, thus creating light, strong wings. 
From these studies, it is possible to draw several conclusions. The first is that corrugated wings delay stalling in high speed flows. Second, the corrugated wing can either act similar to a flat plate or an asymmetrical airfoil in lift generation. Finally, the corrugation is an evolutionary mechanism to reduce the material needed for structurally stiff wings. These conclusions will be used as guidelines for this numerical study, and the objectives will be outlined in the following section. 


\section{Objectives}

The majority of studies carried out have been with scaled models of dragonfly wings from the Aeshna Cyanea's forewing at high Reynolds numbers of beyond $10^{4}$. However for MAV application, the Reynolds number is typically less than $10^{4}$. Because of MAVs potential slow flight speed of $2 \mathrm{~m} / \mathrm{s}$ to $10 \mathrm{~m} / \mathrm{s}$, and their small wings, the flow around the wing is typically laminar. Because at high Reynolds numbers corrugated wings demonstrate many favorable characteristics relevant to MAV designs, it is worthwhile to study the corrugated wing at the low Reynolds number region in which MAVs operate. In this thesis, the aerodynamic characteristics of a corrugated airfoil taken from an Aeshna Cyanea dragonfly is investigated using numerical simulation at the Reynolds number of 1000 . This will give insight into whether or not the conclusions of high lift, and delayed stall concluded by other researchers can still be applied to the lower Reynolds number regime. To further understand the structural implications of the corrugation, a finite element analysis is performed comparing it to a smooth flat plate. 


\section{NUMERICAL METHOD}

\section{A. Navier-Stokes System of Equations}

In this chapter, the governing equations of the physics involved in the numerical simulation will be introduced. First the governing equations of fluid dynamics will be shown and then we derive how they can be adapted for incompressible viscous flow.

The equations governing fluid flow can be derived from the equations of the conservation of

mass, momentum, and energy (Tannehill et al. 1997). These equations can be written in differential form as

$$
\begin{gathered}
\frac{\partial \rho}{\partial t}+\nabla \cdot(\rho \vec{u})=0 \\
\frac{\partial(\rho \vec{u})}{\partial t}+\nabla \cdot(\rho \vec{u} \vec{u})=-\nabla p+\nabla \cdot \tau \\
\frac{\partial\left(\rho e_{t}\right)}{\partial t}+\nabla \cdot\left(\rho e_{t} \vec{u}\right)=k \nabla \cdot \nabla T-\nabla \rho \cdot \vec{u}+(\nabla \cdot \tau) \cdot \vec{u}
\end{gathered}
$$

Where $\rho$ is the density, $t$ is the time, $\vec{u}$ is the flow field velocity vector, $\mathrm{e}_{\mathrm{t}}$ is the total internal energy, $\mathrm{k}$ is the thermal conductivity, $\mathrm{T}$ is the temperature, and $\tau$ is the shear stress. The first of 
the three equations corresponds to the continuity equation of the conservation of mass law applied to a fluid passing through an infinitesimal fixed volume. The momentum equation is the second row, which is Newton's Second law applied to a fluid passing through an infinitesimal, fixed volume. Finally the third row, describes the Energy Equation in terms of the First Law of Thermodynamics.

For low speed flows, it can be assumed that the fluid's density is constant. The constant density can usually be assumed in almost all fluids, because their compressibility is neglected. For gasses with a Mach number lower than 0.3 , the flow is usually considered to be incompressible. If the fluid is assumed to be incompressible and has constant viscosity, then the governing equations can be further simplified in to the following reduced set of equations (Tannehill et al. 1997, Guerrero 2009)

$$
\begin{gathered}
\nabla \cdot \vec{u}=0 \\
\frac{\partial \vec{u}}{\partial t}+\vec{u} \cdot \nabla \vec{u}=\frac{-\nabla p}{\rho}+v \nabla^{2} \vec{u}
\end{gathered}
$$

where $v$ is the kinematic viscosity.

Further simplification can be made by nondimensionalizing the equations. Doing so will provide the conditions upon which dynamic similarity may be achieved for geometrically similar situations. Nondimensionalizing the equations also normalizes values so that they fall between zero and one which also reduces error by making the solution independent of any system of units (Guerrero 2009). Also by normalizing the equations, density can be omitted from the equations since its value would equal one. This form of the Navier-Stokes equations can easily be rewritten in the form

$$
\nabla \cdot(\vec{u})=0
$$




$$
\frac{\partial \vec{u}}{\partial t}+\vec{u} \nabla \cdot \vec{u}=-\nabla p+\frac{1}{\operatorname{Re}_{L}} \nabla^{2} \vec{u}
$$

where viscosity is replaced by the Reynolds number, $R e_{L}$, which is the nondimensionalization of the freestream density $\rho$, velocity $\mathrm{U}_{\infty}$, viscosity $\mu$, and the reference length $L$. The equation for the Reynolds number is as follows

$$
\operatorname{Re}_{L}=\frac{\rho_{\infty} U_{\infty} L}{\mu_{\infty}}
$$

Equation 2.3 can be further altered and expanded into Cartesian coordinates

$$
\begin{gathered}
\frac{\partial u}{\partial x}+\frac{\partial v}{\partial y}+\frac{\partial w}{\partial z}=0 \\
\frac{\partial u}{\partial t}+u \frac{\partial u}{\partial x}+v \frac{\partial u}{\partial y}+w \frac{\partial u}{\partial z}=-\frac{\partial p}{\partial x}+\frac{1}{\operatorname{Re}_{L}}\left(\frac{\partial^{2} u}{\partial x^{2}}+\frac{\partial^{2} u}{\partial y^{2}}+\frac{\partial^{2} u}{\partial z^{2}}\right) \\
\frac{\partial v}{\partial t}+u \frac{\partial v}{\partial x}+v \frac{\partial v}{\partial y}+w \frac{\partial v}{\partial z}=-\frac{\partial p}{\partial y}+\frac{1}{\operatorname{Re}_{L}}\left(\frac{\partial^{2} v}{\partial x^{2}}+\frac{\partial^{2} v}{\partial y^{2}}+\frac{\partial^{2} v}{\partial z^{2}}\right) \\
\frac{\partial w}{\partial t}+u \frac{\partial w}{\partial x}+v \frac{\partial w}{\partial y}+w \frac{\partial w}{\partial z}=-\frac{\partial p}{\partial z}+\frac{1}{\operatorname{Re}_{L}}\left(\frac{\partial^{2} w}{\partial x^{2}}+\frac{\partial^{2} w}{\partial y^{2}}+\frac{\partial^{2} w}{\partial z^{2}}\right)
\end{gathered}
$$

This form provides the simplest discretization and is widely used in numerical models for solving the incompressible Navier-Stokes equations (Tannehill et al. 1997). For incompressible fluids flow with constant viscosity the Navier-Stokes equations have been decouple the energy and density equations. It is now possible to rewrite equation 2.1 into a compact vector form in Cartesian coordinates.

$$
\frac{\partial Q}{\partial t}+\frac{\partial E_{i}}{\partial x}+\frac{\partial F_{i}}{\partial y}+\frac{\partial G_{i}}{\partial z}=\frac{\partial E_{v}}{\partial x}+\frac{\partial F_{v}}{\partial y}+\frac{\partial G_{v}}{\partial z}
$$

where $Q$ is the original vector of spatial variables independent of energy and density 


$$
Q=\left[\begin{array}{l}
0 \\
u \\
v \\
w
\end{array}\right]
$$

$\mathrm{E}_{\mathrm{i}}, \mathrm{F}_{\mathrm{i}}$, and $\mathrm{G}_{\mathrm{i}}$ are the vectors containing the inviscid fluxes in the $x, y$, and $z$ directions and are given as

$$
E_{i}=\left[\begin{array}{c}
u \\
u^{2}+p \\
u v \\
u w
\end{array}\right], F_{i}=\left[\begin{array}{c}
u \\
v u \\
v^{2}+p \\
v w
\end{array}\right], G_{i}=\left[\begin{array}{c}
u \\
w u \\
w v \\
w^{2}+p
\end{array}\right]
$$

$\mathrm{E}_{\mathrm{v}}, \mathrm{F}_{\mathrm{v}}$, and $\mathrm{G}_{\mathrm{v}}$, contain the viscous fluxes in the $x, y$, and $z$ directions and they are given as

$$
E_{v}=\left[\begin{array}{c}
0 \\
\tau_{x x} \\
\tau_{x y} \\
\tau_{x z}
\end{array}\right], F_{v}=\left[\begin{array}{c}
0 \\
\tau_{y x} \\
\tau_{y y} \\
\tau_{y z}
\end{array}\right], G_{v}=\left[\begin{array}{c}
0 \\
\tau_{z x} \\
\tau_{z y} \\
\tau_{z z}
\end{array}\right]
$$

The viscous stress terms $\tau_{\mathrm{xx}}, \tau_{\mathrm{yy}}, \tau_{\mathrm{zz}}, \tau_{\mathrm{xy}}, \tau_{\mathrm{yx}}, \tau_{\mathrm{xz}}, \tau_{\mathrm{zx}}, \tau_{\mathrm{yz}}$, and $\tau_{\mathrm{zy}}$ are also affected by the incompressible assumption. These are defined by the following relationships

$$
\begin{aligned}
\tau_{x x} & =2 \mu\left(\frac{\partial u}{\partial x}\right) \\
\tau_{y y} & =2 \mu\left(\frac{\partial v}{\partial y}\right) \\
\tau_{z z} & =2 \mu\left(\frac{\partial w}{\partial z}\right) \\
\tau_{y x}=\tau_{x y} & =\mu\left(\frac{\partial u}{\partial y}+\frac{\partial v}{\partial x}\right) \\
\tau_{z x}=\tau_{x z} & =\mu\left(\frac{\partial u}{\partial z}+\frac{\partial w}{\partial x}\right)
\end{aligned}
$$




$$
\tau_{z y}=\tau_{y z}=\mu\left(\frac{\partial v}{\partial z}+\frac{\partial w}{\partial y}\right)
$$

We have presented the Navier-Stokes equations in terms of a Cartesian coordinate system. However, many applications require the equations to be expressed in a curvilinear coordinate system that allows the system of equations to be applied to a distorted region of physical space. However, this process will not be covered in this chapter. 


\section{B. Solving the Incompressible Navier Stokes Equations}

This section will give a brief discussion of the numerical method used to solve the governing equations outlined in the previous sections. The method was developed by Henshaw (1994), and Henshaw, Kreiss, and Reyna (1994) for solving the formulation of the incompressible NavierStokes equations on structured overlapping grids. The governing equations will be expressed in Cartesian coordinates for simplicity. In order to transform the governing equations from physical space to computational space, the Cartesian derivates in the governing equations need to be replaced by their transformed equivalent in computational space. Recalling equation (2.2), the initial-boundary-value problem (IBVP) for the Navier-Stokes equation is

$$
\begin{array}{cc}
\frac{\partial \vec{u}}{\partial t}+\vec{u} \cdot \nabla \vec{u}=\frac{-\nabla p}{\rho}+v \nabla^{2} \vec{u} & \text { for } x \in D, t>0 \\
\nabla \cdot \vec{u}=0 & \text { for } x \in D, t>0
\end{array}
$$

with the initial conditions and boundary conditions

$$
\begin{array}{cc}
\vec{u}(\vec{x}, t)=\vec{u}_{0}(\vec{x}) & \text { for } x \in D, t_{0}=0 \\
B(\vec{u}, p)=g & \text { for } x \in \partial D, t \geq 0
\end{array}
$$

In this IBVP, the vector $\vec{x}$ contains the Cartesian coordinates $(\mathrm{x}, \mathrm{y}, \mathrm{z})$ in physical space $P$, and $D$ is a bounded domain in $P \in R^{N}$ (where $\mathrm{N}=1,2,3, \ldots$ ), $\partial D$ is the boundary of the domain $D, t$ is the physical time, vector $\vec{u}$ contains the Cartesian velocities in physical space, $p$ is the pressure, $\rho$ is the fluid density, $v$ is the fluid kinematic viscosity, $B$ is a boundary operator, $g$ is the boundary data, and $\vec{u}_{0}$ is the initial data. This system of equations is called the velocity-divergence 
formulation of the Navier-Stokes equations. Applying the divergence operator to the NavierStokes equations and taking consideration of the continuity equation, a governing equation for the pressure field can be derived. Consequently, the following alternative system of equations called the velocity-pressure formulation can be created (Henshaw 1994, Guerrero 2009)

$$
\begin{array}{cc}
\frac{\partial \vec{u}}{\partial t}+\vec{u} \cdot \nabla \vec{u}=\frac{-\nabla p}{\rho}+v \nabla^{2} \vec{u} & \text { for } x \in D, t>0 \\
\frac{\nabla^{2} \mathrm{p}}{\rho}+\nabla \mathrm{u} \cdot \overrightarrow{\mathrm{u}}_{\mathrm{x}}+\nabla \mathrm{v} \cdot \overrightarrow{\mathrm{u}}_{\mathrm{y}}+\nabla \mathrm{w} \cdot \overrightarrow{\mathrm{u}}_{\mathrm{z}}=0 & \text { for } x \in D, t>0
\end{array}
$$

The corresponding boundary and initial conditions became

$$
\begin{array}{cc}
\nabla \cdot \vec{u}=0 & \text { for } x \in \partial D, t>0 \\
B(\vec{u}, p)=g & \text { for } x \in \partial D, t>0 \\
\vec{u}\left(x, t_{0}\right)=\vec{u}_{0}(x) & \text { for } x \in D, t_{0}=0
\end{array}
$$

Using equations (2.15) and (2.16), an approximate numerical solution is usually sought after in a given domain $D$ with the prescribed boundary and initial conditions, equations (2.17)-(2.19). Equation (2.16) implies that the pressure can be calculated provided the velocity field is known, this is called the Poisson-pressure equation (PPE). The PPE is derived by taking the divergence of the momentum equation (2.11) and using the divergence-free constraint of $\nabla \cdot \vec{u}=0$. The constraint is replaced by the elliptic equation for the pressure. In order for this set of equations to be well defined, an extra boundary condition is required. The extra condition of $\nabla \cdot \vec{u}=0$ for $x \in \partial D$ is added. This ensures the system of equations are equivalent to the original formulation (equations (2.11)-(2.14)). (Henshaw 1994) However, the lack of a proper explicit boundary condition for the PPE causes trouble when implementing the numerical model based on the 
velocity-pressure formulation. To compensate, the use of the normal component, $\hat{n}$, of the momentum equation (2.15), allows us to obtain

$$
\frac{\partial p}{\partial \hat{\mathrm{n}}}=\hat{\mathrm{n}} \cdot\left(-\overrightarrow{\mathrm{u}}_{t}-(\overrightarrow{\mathrm{u}} \cdot \nabla) \overrightarrow{\mathrm{u}}+\nu \nabla^{2} \overrightarrow{\mathrm{u}}\right) \rho \quad \text { for } x \in \partial D, t>0
$$

A divergence term is added in the form of $\alpha_{\mathrm{dc}} \nabla \cdot \overrightarrow{\mathrm{u}}$ to the PPE to suppress the spurious divergence, so that equation (2.16) becomes

$$
\frac{\nabla^{2} \mathrm{p}}{\rho}+\nabla \mathrm{u} \cdot \overrightarrow{\mathrm{u}}_{\mathrm{x}}+\nabla \mathrm{v} \cdot \overrightarrow{\mathrm{u}}_{\mathrm{y}}+\nabla \mathrm{w} \cdot \overrightarrow{\mathrm{u}}_{\mathrm{z}}-\alpha_{\mathrm{dc}} \nabla \cdot \overrightarrow{\mathrm{u}}=0 \quad \text { for } x \in \partial D, t>0
$$

This extra divergence term can be interpreted as a divergence sink or an artificial dampening term, helping to keep the divergence small. The divergence is primarily caused by truncation errors and calculation errors between the interpolation of information between computational domains. The $\alpha_{d c}$ coefficient is usually selected by the user and is discussed in Henshaw (1994), Henshaw, Kreiss, and Reyna (1994). If $\alpha_{d c}$ is properly chosen, it will prevent spurious divergence, and have minimum impact on the calculation accuracy. If it is too small, the system may become unstable, if too large; the system may be over dampened.

Equation (2.15) is discretized into computational space using second-order centered finitedifference approximations. The resulting system of equations can be expressed in a system of ordinary differential equations of the form

$$
\frac{d \vec{u}}{d t}=F(\vec{u}, p, t)
$$

The velocity field can be solved with a given pressure field which is either by solving equation (2.21) or from an initial guess. In order to keep the pressure equation decoupled from the solution of the velocity components, a time stepping scheme must be chosen for the velocity 
components that involves the pressure from the previous time step. This is called a split-step scheme. Then the equations are solved using a semi-implicit multistep method that uses a CrankNicolson scheme for the viscous terms and second-order Adams-Bashforth predictor-corrector for the convective terms and pressure. (Guerrero 2009)

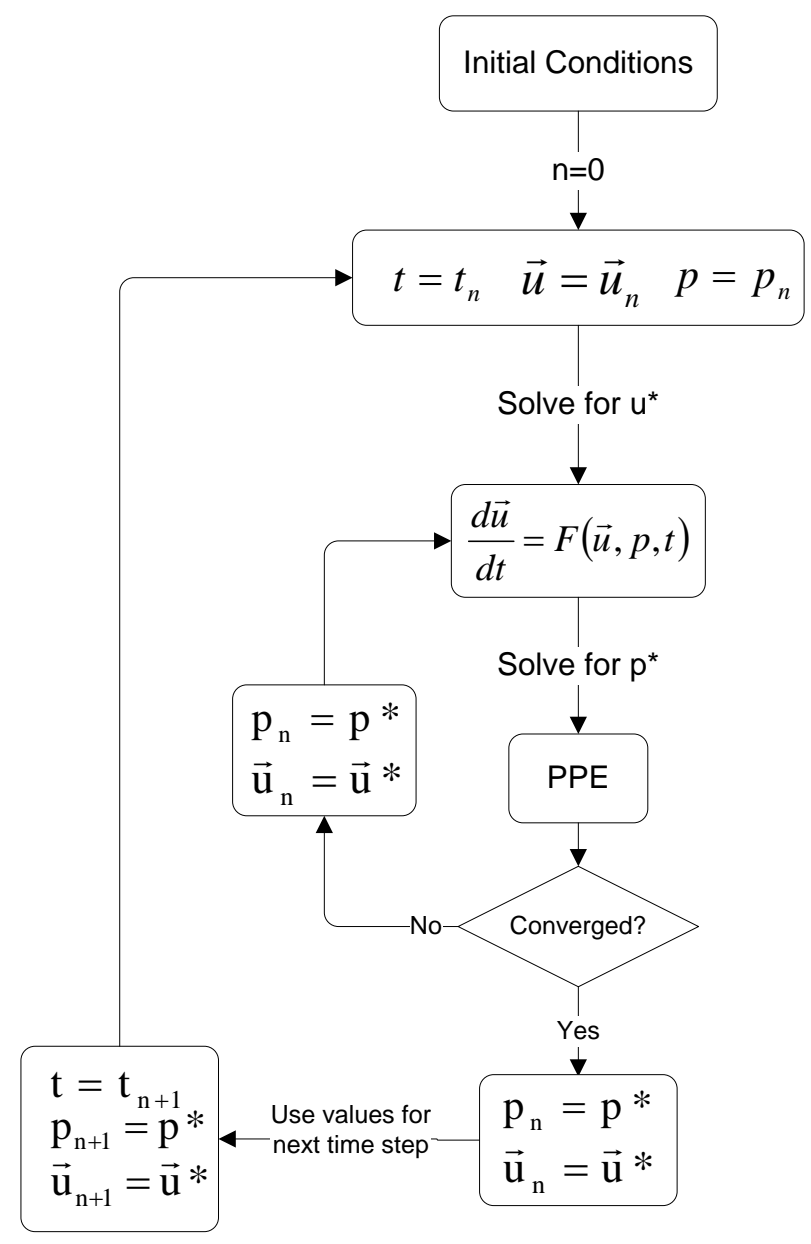

FIGURE 4 - Process diagram of split-step scheme of the Navier-Stokes equation

The entire process is illustrated in Figure 4, where an initial condition of $\mathrm{U}_{0}$ and $\mathrm{p}_{0}$ are inputted at time zero. $U^{*}$, an intermediate velocity value, is solved using equation (2.22), which then is inputted into the PPE, equation (2.21). An intermediate pressure, $\mathrm{p}^{*}$, is solved, and $\mathrm{U}^{*}$ and $\mathrm{p}^{*}$ checked for convergence, if not, both are inputted back into equation (2.22). Once 
converged, $\mathrm{p}^{*}$ and $\mathrm{U}^{*}$ become the solution to the pressure and velocity at that time step, and then are used to initialize the next time step. 


\section{Boundary Conditions}

Other boundary conditions can be imposed on the incompressible Navier-Stokes equations than previously discussed. Using the appropriate conditions is paramount in solving any governing equations. The types of boundary conditions are dependent on the type of flow, and geometry for a selected problem. Once these two criteria have been determined, it is possible to numerically solve the velocity-pressure equation using the Overture framework coupled with the PETSc $^{2}$ library. Inside the Overture package, Cgins is used to solve the velocity-pressure equation discretized across an overlapping grid. Cgins offers easy implementation of boundary conditions, and offers the following types (Henshaw 2006)

$$
\begin{aligned}
& \text { No-Slip }=\left\{\begin{array}{cc}
\overrightarrow{\mathbf{u}}=g & \text { Velocity specified } \\
\nabla \cdot \overrightarrow{\mathbf{u}}=0 & \text { Zero divergence }
\end{array}\right. \\
& \text { Slip wall }=\left\{\begin{array}{cl}
\hat{\mathbf{n}} \cdot \overrightarrow{\mathbf{u}}=g & \text { Normal velocity specified } \\
\partial_{\hat{n}}(\hat{t} \cdot \overrightarrow{\mathbf{u}})=0 & \text { Normal derivative of tangential } \\
\nabla \cdot \overrightarrow{\mathbf{u}}=0 & \text { velocity is zero } \\
& \text { Zero divergence }
\end{array}\right. \\
& \begin{aligned}
\text { Inflow with velocity } \\
\text { given }
\end{aligned}=\left\{\begin{array}{cl}
\overrightarrow{\mathbf{u}}=g & \text { Velocity specified } \\
\partial_{\hat{n}} p=0 & \text { Normal derivative of the pressure } \\
\text { zero }
\end{array}\right. \\
& \text { Outflow }= \begin{cases}\text { extraplotate } \quad \overrightarrow{\mathbf{u}} & \text { Velocity specified } \\
\alpha p+\beta \partial_{\hat{n}} p=g & \text { Mixed derivative of } p \text { given }\end{cases} \\
& \begin{aligned}
\text { Dirichlet boundary } \\
\text { condition }
\end{aligned}= \begin{cases}\overrightarrow{\mathbf{u}}=g & \text { Velocity specified } \\
p=P & \text { Pressure given }\end{cases} \\
& \text { Symmetry }=\left\{\begin{array}{cl}
\hat{\mathbf{n}} \cdot \overrightarrow{\mathbf{u}}: \text { odd }, \hat{\mathbf{t}} \cdot \overrightarrow{\mathbf{u}}: \text { even } & \text { Vector symmetry } \\
\partial_{\hat{n}} p=0 & \text { Normal derivative of pressure zero }
\end{array}\right.
\end{aligned}
$$

\footnotetext{
${ }^{2}$ http://www-unix.mcs.anl.gov/petsc/petsc-as/
} 


\section{OVERLAPPING GRIDS}

\section{A. Types of Grid Generation}

In the last chapter, the governing equations for low speed incompressible flows were discussed. Developing the equations is only part of the solution for solving flow fields in complex domains. A grid or mesh must be generated to allow for the governing PDEs to be spatially discretized. Grid generation can be described as breaking a continuous domain into smaller domains. There are three main types of grids: body-fitting structured grids, unstructured grids, and Cartesian grids (Thompson et al 1998).

Body-fitting structured grids are constructed of repeating rectangular blocks, thus where it derives its name. In structured grids, the domain is rectangular in shape where the interior grid points are distributed along the grid lines. This allows for easy identification of each grid point in reference to the grid lines. Figure 5 illustrates a simple structured grid fitted to an annulus inside a square domain. Structured grids also provide a major advantage over other types of grid generation. They allow for a high degree of user control. The user has total control how the mesh is generated and positioned. This enables the user to place control schemes that concentrate 
points in regions of complex flow interactions, such as boundary layers. Because the grid is user controlled, this allows for the grid to be developed in a fashion that maximizes the computational efficiency of the grid, allowing for faster, and more accurate computed PDE solutions.

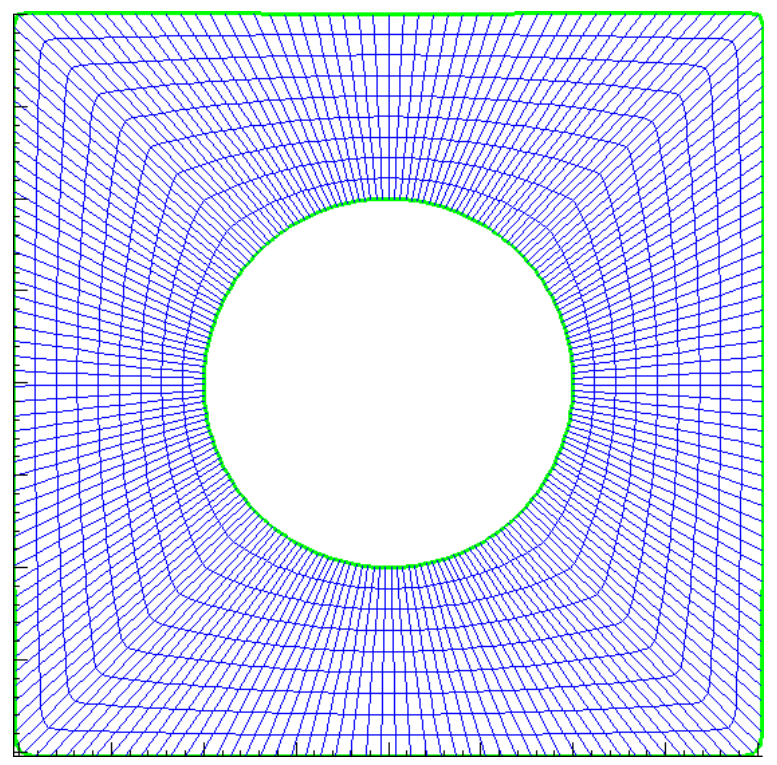

FIGURE 5 - A structured grid meshed between an annulus and square.

The main disadvantage to structured grids occurs when a multi-block method has to be employed. When complex geometries need to be blocked to non-trivial boundaries, the user can break the domain into several sections. Each section has independently generated structured grids inside of them, and then a grid is generated between the sections to connect them. Figure 6 shows the same annulus and square domain as in Figure 5 but created with multiple structured grids. One grid generated around the annulus, to allow for a more uniform grid around it, while four other structured grids are meshed to fit the square. While this gives the user more control in generating grids for complex geometries, connecting the grids can be difficult and tedious. Because of this problem, structured grid generation sometimes requires a high level of skill from the user, and sometimes takes weeks to create an optimized grid. 


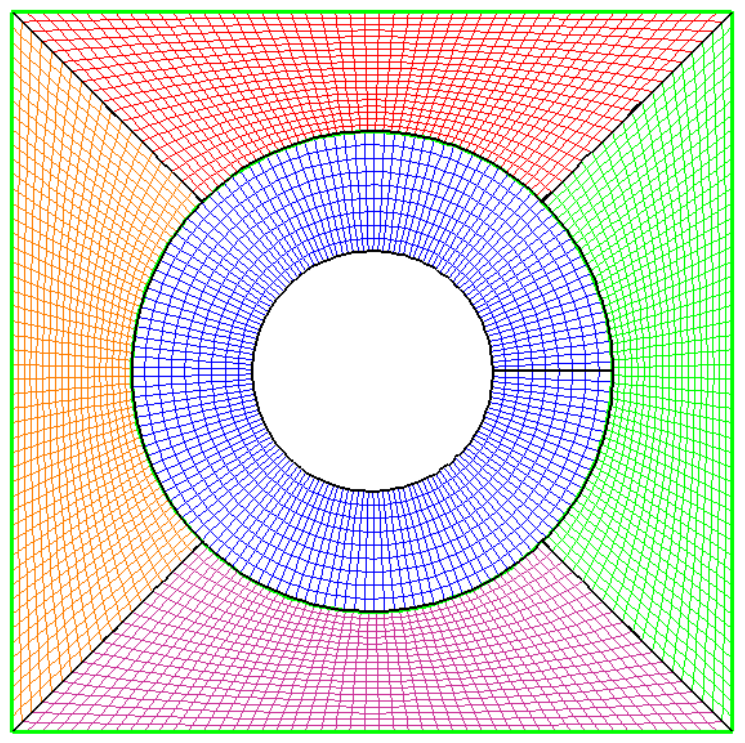

\section{FIGURE 6 - A multiblocked structured grid.}

Unstructured grids resolve the some of the problems associated to structured grids. Instead of using rectangular elements, unstructured grids use triangular elements. A generated unstructured grid has no recognizable pattern as a structured grid has, and this is why it is called unstructured. One advantage to unstructured grids is that elements are much easier to position to fit the requirements of the domain. Another is the process of grid generation can be automated to a fairly large degree, requiring very little user input. The user does not have to worry about laying out properly generated structured sections, and then connecting them. This allows inexperienced users to quickly generate grids in a matter of minutes or hours as opposed to days or weeks. Figure 7 shows an example of the annulus and square meshed with an unstructured grid. 


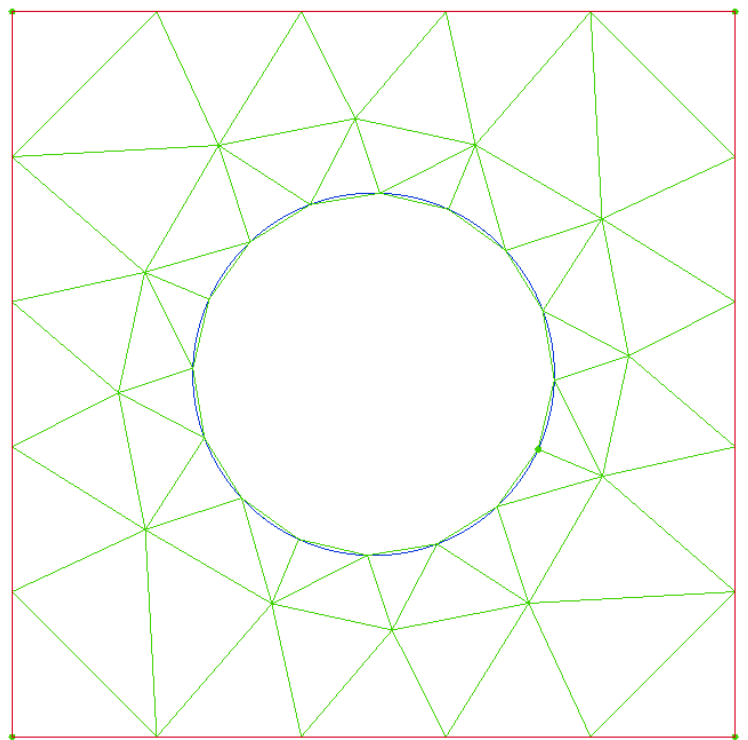

FIGURE 7 - Unstructured annulus and square mesh (Dou et al 1998)

The major disadvantage to unstructured grids is the lack of controllability. Usually the user has very limited control in how the grid is generated and positioned. Also the triangular elements do not stretch well, the grids usually appear uniform. Unlike structured grids, unstructured grids cannot be locally refined to meet the needs of the user. Thus often times, regionally grid densities have to be increased as opposed to local increases. The last drawback is that unstructured grids take significantly more time and memory to solve. This is because unstructured grids do not have an easy grid point reference system like the structured grids.

Both methods can be referred to as body-fitted grid generation. This means the blocks near the geometries surface must conform to that surface. As a result the surface mesh can conflict with itself, and the requirements of the user and the flow field. To resolve this issue, a resurgence of interest in Cartesian grids has come about in the last decade.

The Cartesian method abandons the use of body-fitted grids all together. Instead it uses a Cartesian background grid. The geometry is cut out of the grid leaving a set of irregularly shaped cells on the boundary of the geometry. Cartesian grids use a second method called adaptive mesh 
refinement (AMR). (Aftosmis 1997) Without this automatic refinement, the Cartesian grids would lack the ability to efficiently resolve fluid and geometry features. This works by actively adding more uniform grid points in areas of where the flow field is not converging until the flow field is resolve to a satisfactory degree. This is a major advantage to Cartesian grid generation, because the grid generation is fully automated. Since there are no body-fitted grids, the grid quality and refinement can be fully automatically. Although because of the geometry is not smooth but, almost stair case like (Guererro 2009), this puts a greater burden on the flow solver. Figure 8 illustrates how the annulus and square example would look. The annulus loses its smooth edge, however a finer Cartesian grid could reduce the "staircase" effect. However, highly efficient and accurate flow solvers can be implemented easier using this technique resulting in better solutions. The single drawback of Cartesian grids is that their use is currently restricted to inviscid or low Reynold number flows (Aftosmis 1997).

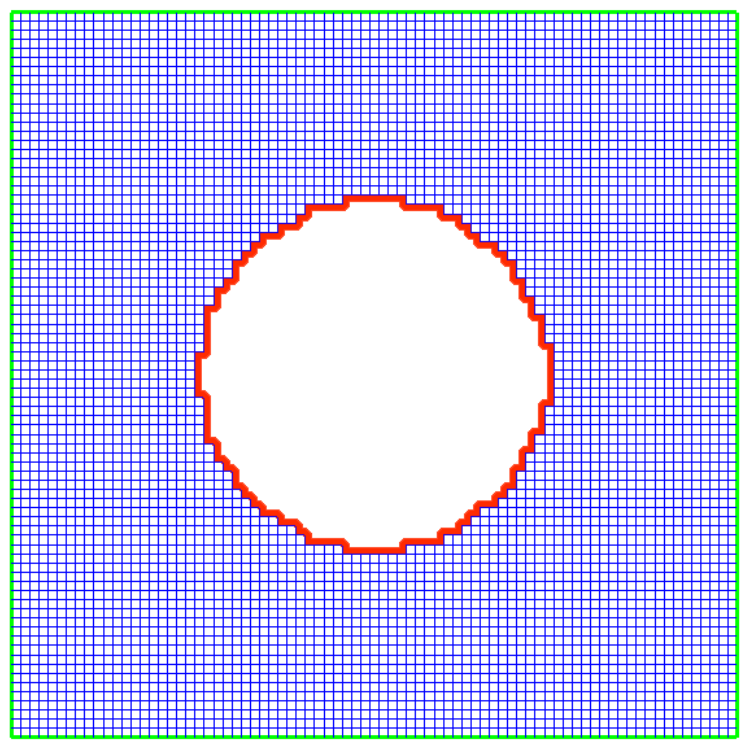

FIGURE 8 - Cartesian grid of annulus and square. 
These three approaches discussed are the most basic forms of grid generation. The next section, a newer form of grid generation methodology based on structured grids that resolve many of its inheriting problems will be described. 


\section{B. Overlapping Structured Grids}

Overlapping structured grids, also known as composite grids or Chimera grids, provide a more flexible method of grid generation, yet still provide efficient spatial discretization for numerically solving the governing PDEs. The overlapping grid method consists of generating a set of body-fitted structured grids that completely cover the physical domain that is being modeled and overlap where they meet. Like previous figures, Figure 9 illustrates how overlapping grids usually function. A structured grid is body-fitted around the annulus, and then overlaid onto of a uniform background grid. The governing PDEs are solved separately on each grid and then connected to each other through interpolation in the overlapping areas. With the ability to define each of the components individually, the grids around them can also be generated individually also.
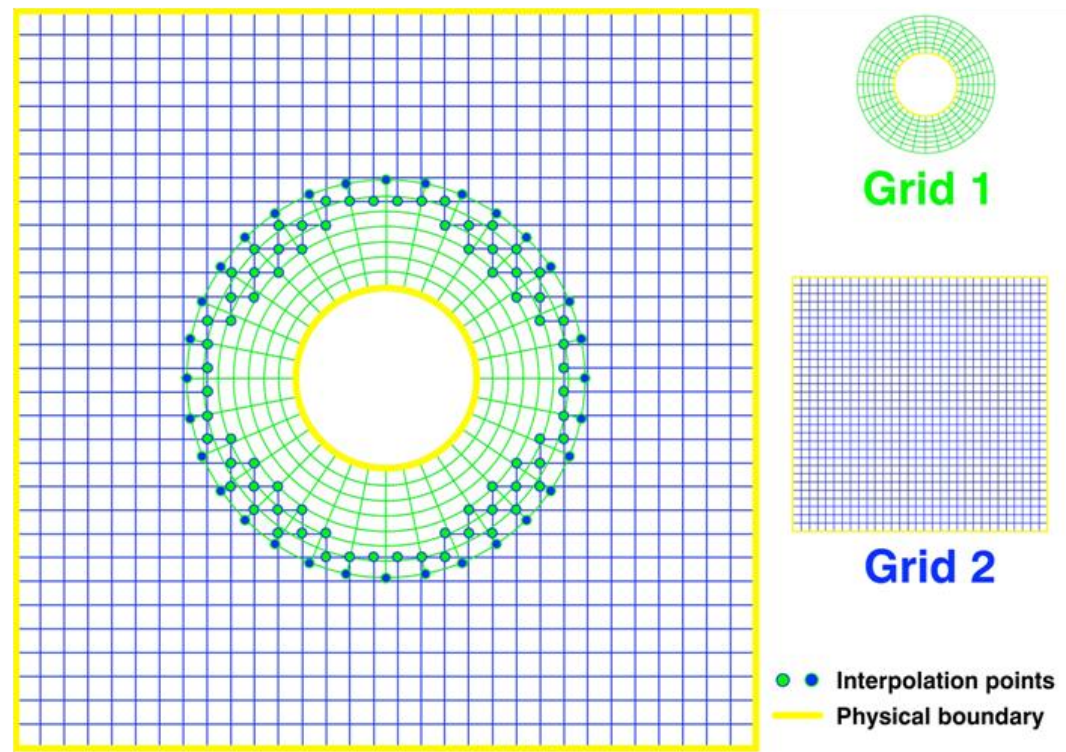

FIGURE 9 - Example of Overlapping Structured Grid system (Guerrero 2009, Henshaw 1998) 
Normally body-fitted grids are used near the geometry of the model, while one or more Cartesian grids are used in the background to handle the rest of the domain. Overlapping grids were originally developed to address complex geometry, multiple body motion, and to resolve fine-scale flow features through the use of AMR. The generated grids can be easily altered and reproduced nearly instantaneously. This allows for multiple iterations of the same grid to be simulated with different parameters with ease (Henshaw 2003). This is done by only modifying the grid of interest and reoverlapping the grids, as opposed to modifying the entire domain grid structure. Through the use of structured grids coupled with Cartesian grids, a method has been developed that is optimized the spatial discretizations has lead to a highly efficient method in computer time and memory expense. (Henshaw 2003)

While the overlapping grids method has been in use for some time now, it hasn't been of much interest until recently. It was originally described by Volvov in 1966. The method was further developed and promoted by Starius in 1977. Even the method is fairly old; it was not in introduced to the CFD community until the early 1990s. Steger et al. (1983) and Benek et al. (1985); and it has been further developed by Meakin and Suhs (1989), Chesshire and Henshaw (1990) and Noack et al. (2004). This approach is now recognized as an attractive method for solving problems with complex geometries and moving bodies. (Guerrero 2009)

As with all methods, overlapping grids have a few disadvantages. The algorithms to generate the overlapping grids can be far more complex. Mistakes can be easily made in setting grid boundary conditions or overlapping priorities. This makes grid generation an iterative process. If proper planning in the generation process is not done, the overlapping grids can lead to poor interpolation results between grids, leading to increased computational time and poor solution 
convergence. However, if good generation practices are established, many problems can be avoided all together, and grids can be generated fairly quickly. In addition to added complexity, the flow solvers used must be able to handle interpolation across multiple overlapping grids. The data structures required to connect overlapping grids can cause problems if the flow solver adequately programmed correctly. However, even though the process can be more complex compared to purely structured, or unstructured grid domains, the gain in computational efficiency and the ease of altering established grids makes the method worthwhile.

In the next section, it will be discussed how Ogen (Henshaw 2005), a tool in the Overture ${ }^{3}$ package, determines how different grids will interpolate with each other, by eliminating grid points in regions of excess overlap.

\footnotetext{
${ }^{3}$ https://computation.llnl.gov/casc/Overture/
} 


\section{Overlapping Grid Assembly}

In this section, it will be discussed how Ogen, the grid generation tool inside of Overture, assembles overlapping grids. The assembly of overlapping grids consists of three major parts. The first step is the definition of the geometer and grid component generation by the user. The second step consists of grid generation tool detecting all grid points to be removed. The final step, is locating all grid points to be used for interpolation on the edge of the removed points. Only a brief discussion will be give, on how the algorithm works, for a more detailed discussion, the process is well documented in Henshaw's (2005) paper.

To properly describe the major steps and its corresponding sub steps, first a set of component grids and boundary conditions must be defined. Figure 10 illustrates the two component grids that will be used in this example. The annulus, $G_{2}$, is an annulus with a structured body-fitted grid, and $G_{1}$, is a Cartesian background grid.
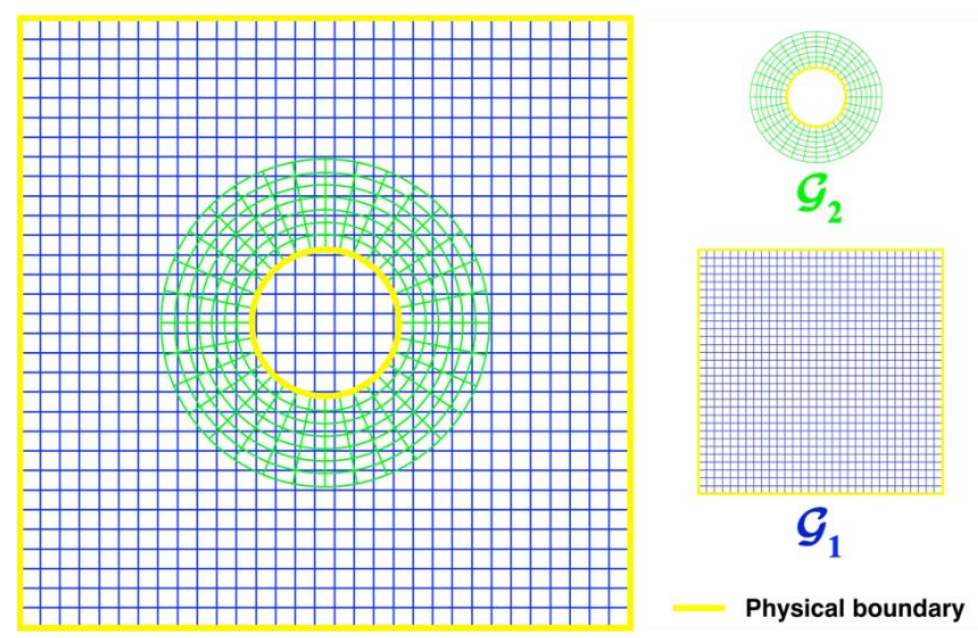

FIGURE 10 - Component grids a Cartesian grid, $G_{1}$, and a body-fitted structured grid around an annulus, $G_{2}$ (Guerrero 2009, Henshaw 1998) 
The next stop begins with the software marking the hole boundaries and removing exterior grid points. This process itself has two-substeps. Firstly, each physical boundary, points are found that lay inside, or outside of other physical boundaries. Therefore, the algorithm will see grid points from $G_{1}$ are laying inside of $G_{2}$, and marked as unused points. After this substep, the unused points will be bounded by a boundary of interpolation points as illustrated in Figure 11. Once this is down, all unused points will be removed. This completes the second step, and the algorithm has created what is known as a Chimera hole (Henshaw 2005).

Interpolation points Unused points

Physical boundaries

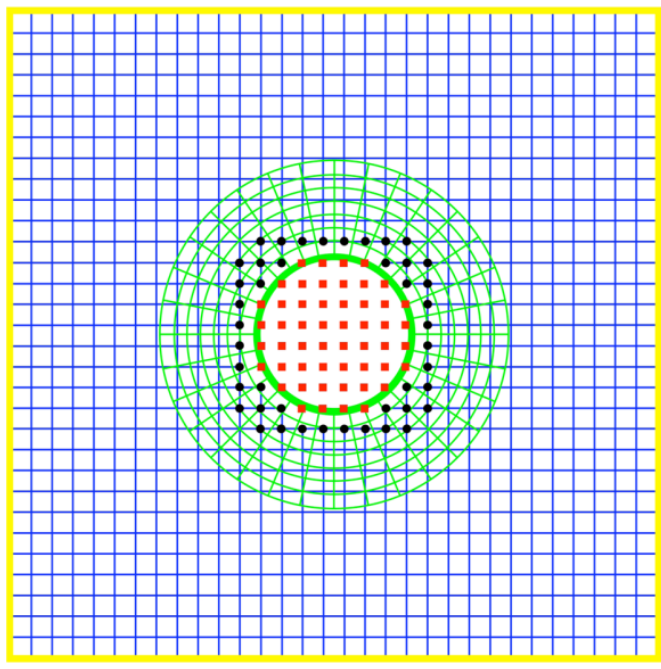

FIGURE 11 - Identifying unused, and interpolation points (Henshaw 1998, Guerrero 2009)

The final step finds and classifies all valid interpolation points. Here, the points on the physical boundaries and interpolation boundaries are collected into a list of interpolation points. Then points are labeled using improper interpolation. A point is said to be improper if it lies inside of another grid. Since all points lie within the domain, they all must interpolate from some other grid. From these improper points, proper points must be then classified. Proper points are 
points that lie along the lines of the two grids being overlapped. These lines will be used for implicit or explicit interpolation for the flow solvers (Henshaw 2005). Once this done, an overlapping grid system has been created. Figure 12 shows what the newly formed system looks like.

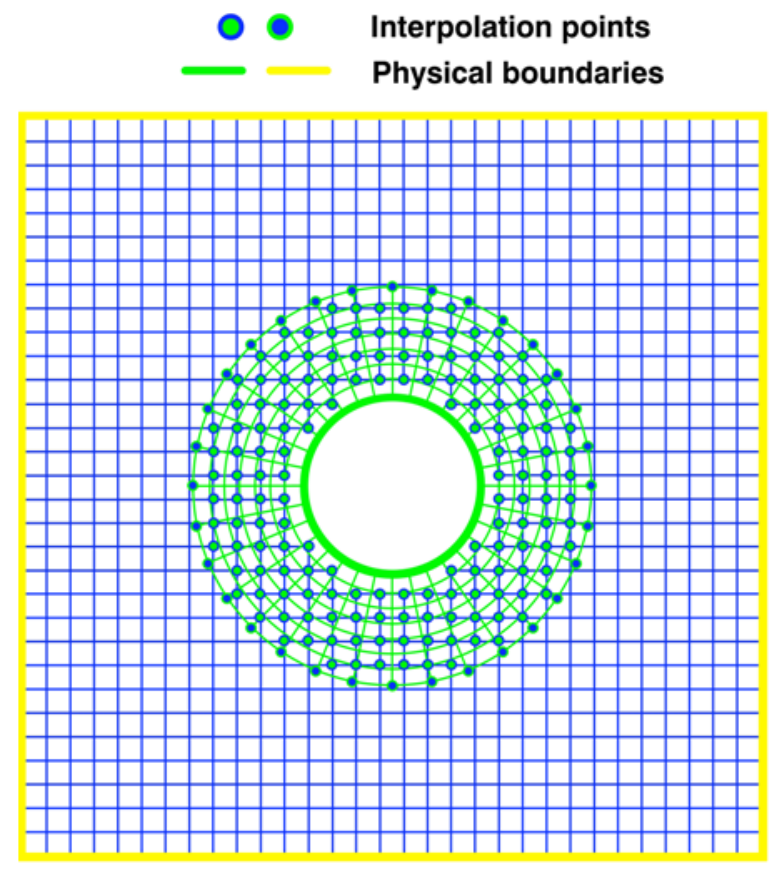

FIGURE 12 - Completed Overlapping grid system (Guerrero 2009, Henshaw 1998)

An additional set can be performed at this stage called trimming. Basically, it reduces the amount of overlapped between the two grids. This is done by removing any interpolation point deemed not needed. The amount of overlapped removed is usually specified by the user or a minimum overlap requirement preset in the algorithm. The final trimmed overlapping grid can be seen in Figure 13. 


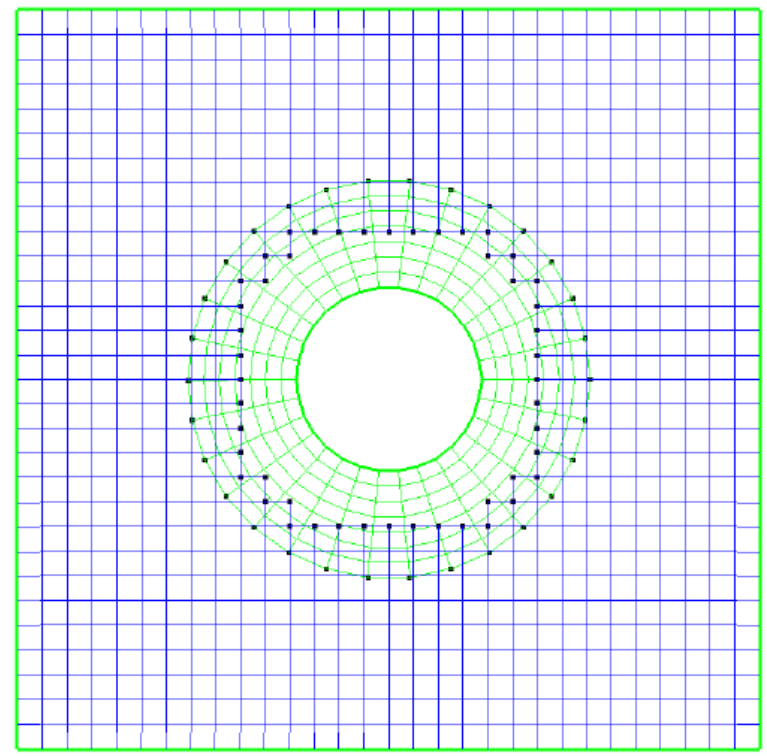

FIGURE 13 - Trimmed overlapping grid (Guerrero 2009, Henshaw 1998)

This completes the major three steps in constructing an overlapping grid. A forth step is also usually completed which checks the consistency of the system to see if it satisfies all requirements. If not, all points that fail to satisfy the requirements will be marked, and outputted as a graphic for troubleshooting. 


\section{Geometry Selection}

To properly begin this study, it must be discussed where the selected geometry of the corrugated profile and the flat plate originated from. Many researchers have tested a variety of corrugated profiles, but there are three prominent profiles usually tested. Figure 14 illustrates the three profiles.

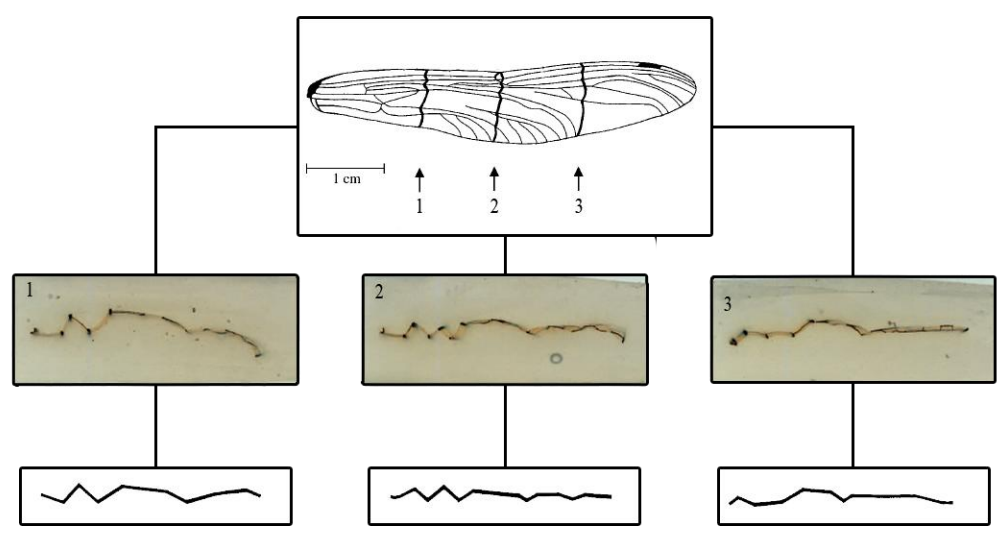

FIGURE 14 - Aeshan Cyana cross section profiles (Okamota 1996, Kesel 2000, Murphy 2009)

Each profile was taken from a cross section of the forewing of an Aeshan Cyana dragonfly at $30 \%, 50 \%$, and $80 \%$ of the wing span. From these three cross sections, an outline was traced. Typically these three profiles are chosen because each offers a different degree of corrugation and leading edge orientation. Usually the $50 \%$ span profile is favored among the three. This is because it has a neutral leading edge. This allows researchers to solely test the effects of corrugation, while eliminating any effect an upward or downward leading edge may have on the overall aerodynamic performance of the profile.

The chosen profile is then generally used as a guideline for building an airfoil for experimental or numerical study. Murphy et al. (2009) took the 50\% span profile and created a 
much thicker profile, Figure 15, for a high Reynold's number wind tunnel study. This profile was chosen for this study for similar reason as listed above.

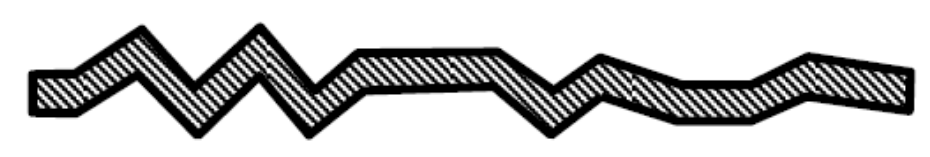

FIGURE 15 - Corrugated profile created by Murphy (2009).

Using Murphy's profile provided an excellent basis to create the profile for the numerical study. It will be discussed later on how this geometry was translated into Overture and how a structured grid was generated around it.

The geometry selection of the flat plate is normally dictated by two procedures: choosing a profile thickness and smooth or sharp leading edges. The profile thickness, $t_{a} / c$, is the ratio of the thickest part of an airfoil's profile divided by its chord length. It was chosen that the flat plate would have a thickness of $2 \%$ and have rounded leading and trailing edges. Sharp edges can be notoriously difficult to generate body fitted grids, thus this would eliminate and difficulties. 


\section{E. Corrugated Profile Grid Generation}

To produce an accurate corrugated profile virtually, a profile taken from Murphy et al. (2009) was used to generate a system of coordinate points. A program called Precision Image Digitizer (PID, Figure 16) was used to create a list of points. Each corner of the wing was marked, and a Cartesian coordinate for the mark was recorded.

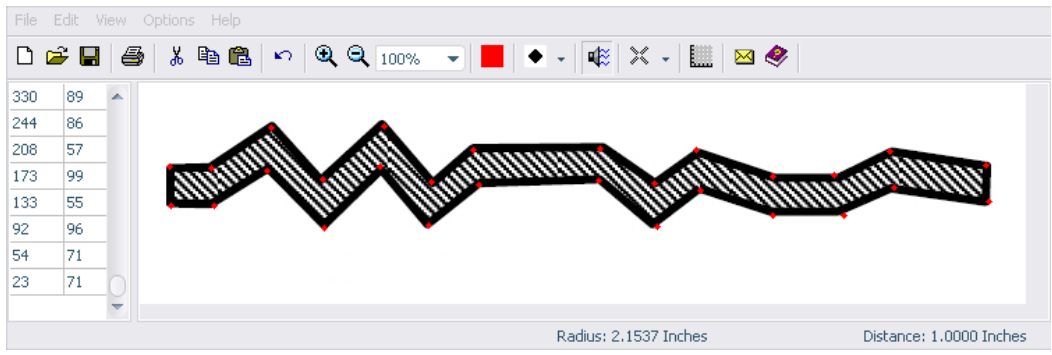

FIGURE 16 - Image of the PID software

Using the coordinates provided by PID, a spline mapping was generated in Ogens, the grid generator tool of Overture. Unfortunately, due to limitations of the spline mapping in Overture, sharp corners were unable to be achieved. The primary reason was due to the lack of coordinate points used. The shape of the corrugated wing was not well defined enough, causing large radius filets at the corners to be produced. This effect was not desired because the sharp corners of the corrugation are hypothesized to be beneficial to the wing's performance (Murphy et al. 2009), and the original shape had to be preserved as much as possible. The coordinate density was increased, and the original shape was achieved, Figure 17. Corners were still rounded, but the effect could be diminished by further increased coordinate density.

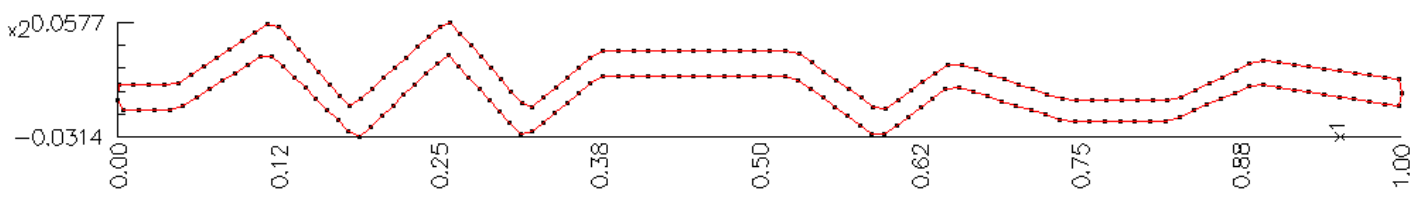

FIGURE 17 - Spline mapping of the corrugated profile 
For Ogen to properly build a group of grids, the corrugated wing spline mapping must be mapped to a structured grid. Give the corrugated profile's highly irregular shape, creating a single body fitting grid proved to be challenging. In order to remedy this, a hyperbolic grid generation function was employed. Typically a structured grid is grown from the surface contours of a spline mapping in the normal direction. Due to the highly irregular features, this would cause grid blocks to intersect and interfere with one another as illustrated in Figure 18.

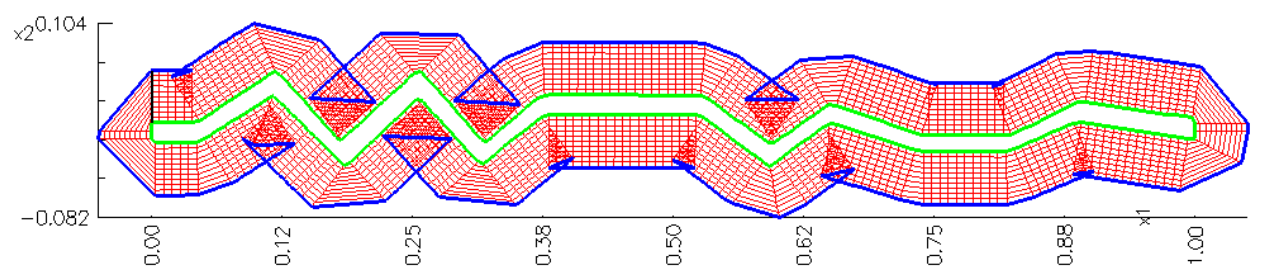

FIGURE 18 - Structured grids grown in the normal direction of surface

Hyperbolic mapping generates a structured grid by marching along the tangential direction of the surface contour of a spline mapping. This method sometimes creates highly non-uniform grids. However, this method also allows for a high degree of user controllability. The user is able to control the minimum and maximum cell size, and stretch cell density into areas of importance.

Figure 19 illustrates the resultant of the hyperbolic grid function with very little user input. As it can be seen the grid is extremely coarse in some areas, very fine in others, and highly irregular all over. Typically it is preferred to have a fairly uniform cell size across the grid, and have the cell size decrease as it approaches the boundary of the surface. Having such an irregularity in the grid's construction as in Figure 19 will sometimes will cause computational errors or increases computational time. By applying user controls, the generated grid can be highly refined as in Figure 20. 


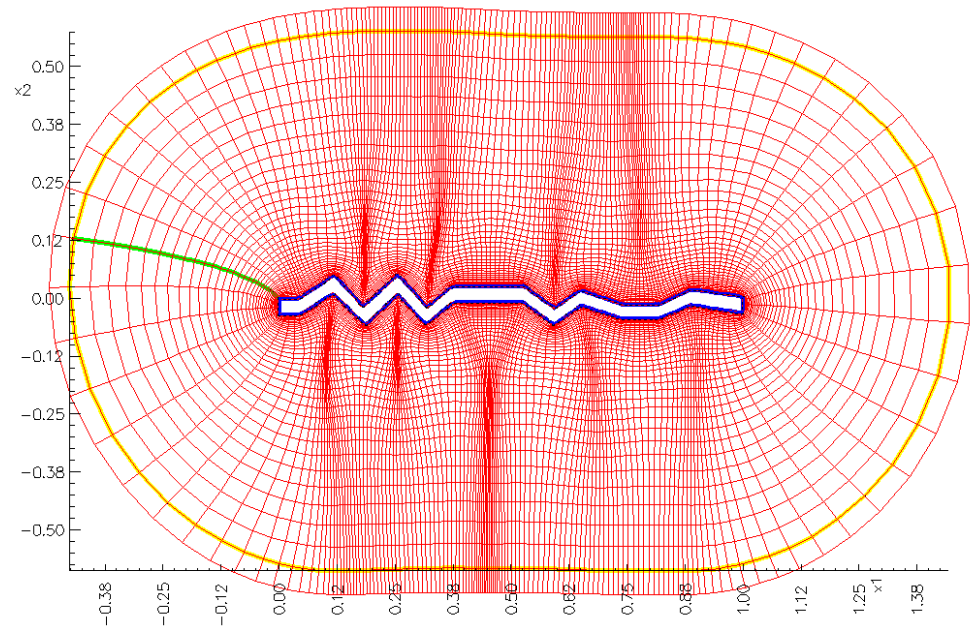

FIGURE 19 - Hyperbolic grid generation no user control

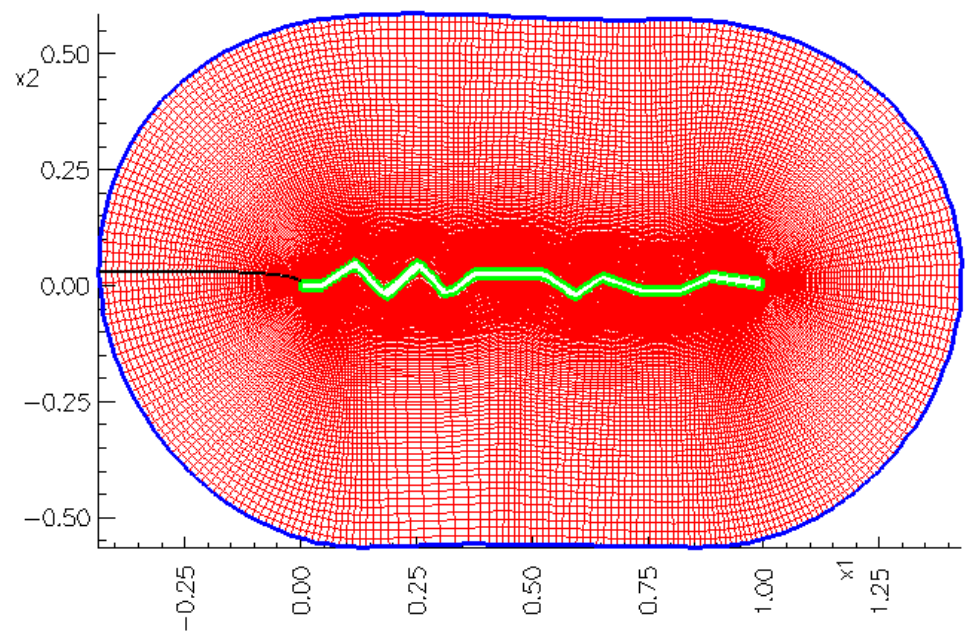

FIGURE 20 - Hyperbolic grid generation with user control 


\section{F. Flat Plate Grid Generation}

The flat plate grid generation process is the same process used for the corrugated profile. The only difference in the procedure is the coordinate data for the flat plate. The coordinates for the flat plate were easily generated, and a spline mapping was produced from them as shown in Figure 21.

\section{FIGURE 21 - Flat plate spline mapping}

While the geometry of the flat plate is vastly simpler than the corrugated profile, the hyperbolic grid generation method was still employed. Other methods could be used, but the user controllability of the hyperbolic function makes it a good tool. Figure 22 shows the structured grid generated for the flat plate.

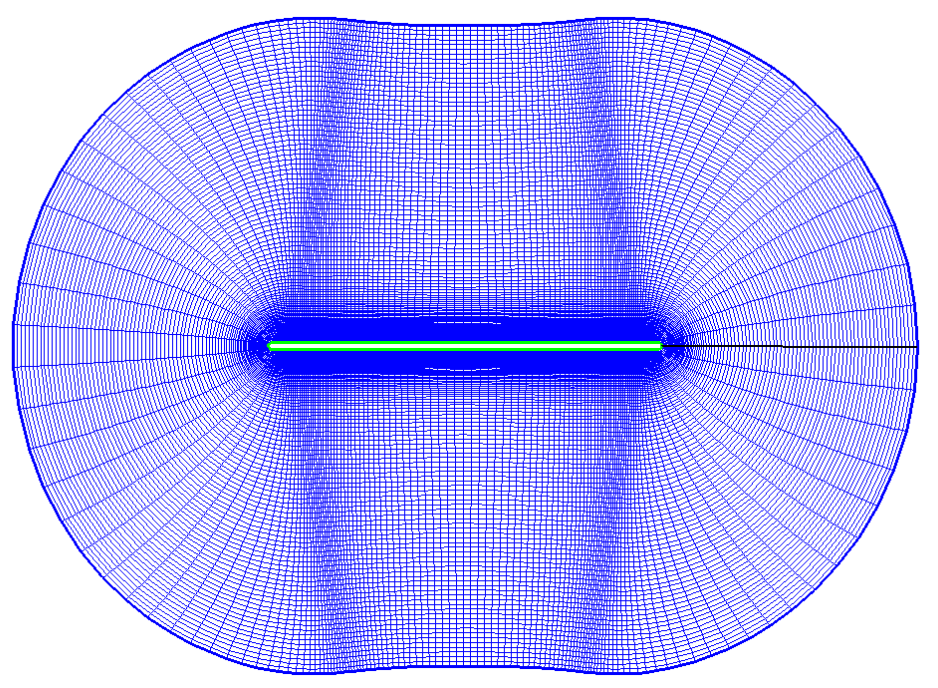

FIGURE 22 - Hyperbolic grid of flat plate 


\section{G. Computational Grid Domain}

The computational domain grid, or the "background grid," is a simply Cartesian grid. The dimensions of the background grid were made so that the boundaries were at least 5 chords away from all surfaces of the airfoil. This was referred to as a 5 chord by 5 chord domain $(5 \mathrm{c} \times 5 \mathrm{c})$. The domain then had 200 lines in the $\mathrm{X}$ and $\mathrm{Y}$ direction. The justification of the dimensions of the background grid will be discussed in the next chapter. The airfoil grids were then overlapped at the center of the background grid. Thus a mild grid stretching was applied to the background grid, so the majority of the grid points lie in the center channel of the background. This allows for minimum grid points to be used in the grid, will increasing the number of grid points used in areas of interest and for interpolation. Figure 23 illustrates the background stretching.

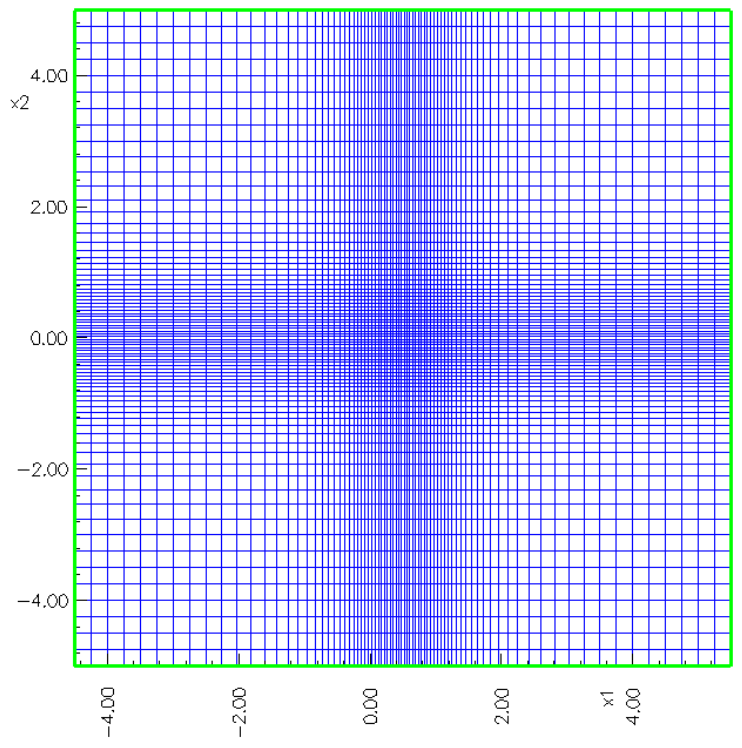

FIGURE 23 - Background grid stretching 


\section{CODE VALIDATION AND COMPUTATIONAL SETUP}

In order to insure time efficient accurate results, a code validation and grid sensitivity analysis was preformed. The code validation would simply show that the code yields accurate results, and the grid sensitivity analysis would show the required grid densities and domain size to insure accurate results. The grid densities of the airfoil grids were systematically varied to see the changes on the coefficient of lift, while the domain size was investigated to minimize blockage effects. While it is generally assumed increasing grid densities yield more accurate solutions, increasing the grid density also exponentially increase computational time. Therefore, it was important to find a balance between computational accuracy and solution time.

\section{A. Code Validation}

In order to accurately determine the required grid densities for each airfoil, and the flow solver must first be validated. The easiest validation method to perform is a uniform flow of varying Reynolds numbers across a circular cylinder. This particular experiment has been thoroughly studied, and documented providing a means to compare the flow solver's results to recorded data from other researchers. 


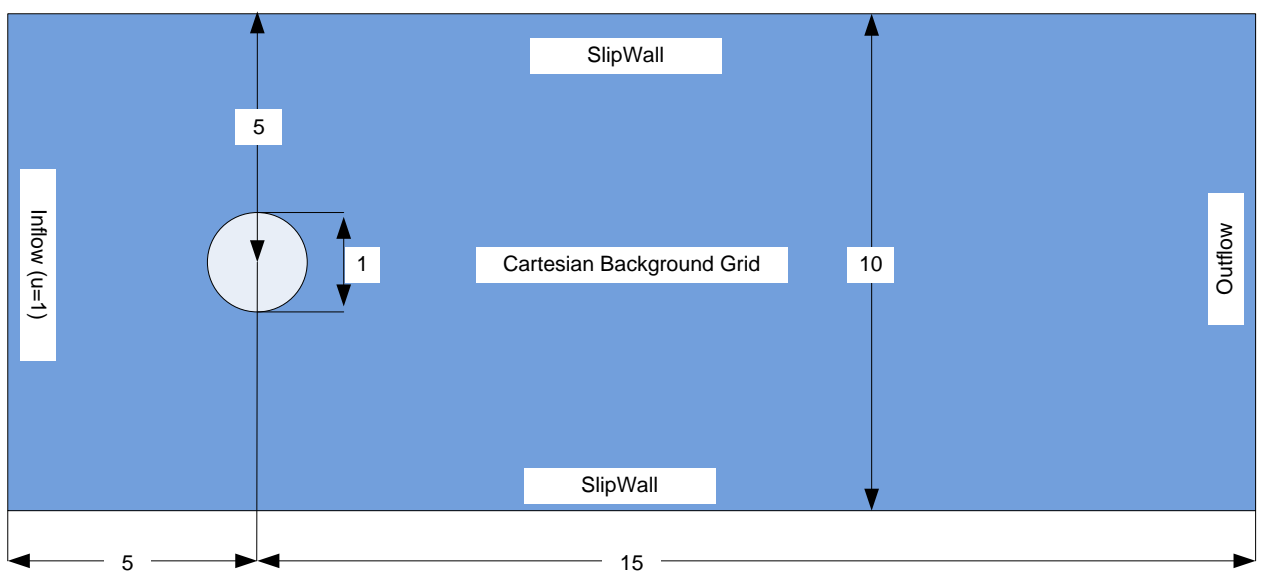

FIGURE 24 - Overlapping grid layout used for code validation

The overlapping grid system used in the validation is shown in Figure 24. The figure also details the dimensions, and boundary conditions used of the computational domain. The Reynolds number was based on the diameter of the cylinder, the kinematic viscosity and the inflow velocity. By varying the kinematic viscosity, the flow solver was tested at Reynolds numbers of 20,40,100, and 200. Table 1 lists the numerical results of the coefficient of drag compared to published data. At Reynolds numbers greater than 50, the flow becomes unsteady and the " \pm " denotes the amplitude of the drag oscillations It was found that the flow solver produced results within the published range confirming the validity of the flow solver.

\begin{tabular}{lcccc}
\hline \hline & \multicolumn{5}{c}{$\mathbf{C}_{\mathbf{D}}$ for Reynolds Number } \\
Reference & $\mathbf{2 0}$ & $\mathbf{4 0}$ & $\mathbf{1 0 0}$ & $\mathbf{2 0 0}$ \\
\hline Calhoun and Wang (2002) & 2.19 & 1.62 & $1.35 \pm 0.014$ & $1.17 \pm 0.058$ \\
Choi et al. (2007) & --- & --- & $1.34 \pm 0.011$ & $1.36 \pm 0.048$ \\
\hline Guerrero (2009) & 2.2013 & 1.6208 & $1.39 \pm 0.012$ & $1.41 \pm 0.098$ \\
Kim and Choi (2005) & --- & 1.501 & 1.336 & --- \\
\hline Posdziech and Grundmann (2006) & 2.07 & 1.54 & 1.35 & 1.35 \\
Russel and Wang (2003) & 2.13 & 1.6 & $1.38 \pm 0.007$ & $1.29 \pm 0.022$ \\
Tritton (1959) & 2.22 & 1.48 & --- & -- \\
Numerical Results & 2.23 & 1.59 & $1.42 \pm 0.007$ & $1.37 \pm 0.030$ \\
\hline \hline
\end{tabular}

Table 2. Comparison of $\mathrm{C}_{\mathrm{D}}$ with published results at varying Reynolds numbers 


\section{B. Domain Size}

Before testing each airfoil grid, the background grid must be properly sized first. In a recent study, Granlund et al. (2010) showed the effects of small domains sizes have on coefficient of lift in water channels. They found the coefficient of lift increased by a factor of two when the domain was halved in size by doubling the chord length of the airfoil. This effect however was not prominent until high angles of attack. The problem primarily stemmed from no-slip wall effects inside of water tunnel. This shows that if a domain was not of sufficient size, the velocity of the fluid at the center of the channel would be sufficiently higher than at the entrance thus increasing lift. Also at high angle of attack, the airfoil can create blockage effects which further exacerbate the problem. In numerical simulation it is possible to mitigate this effect by setting the top and bottom walls to inflow boundaries.

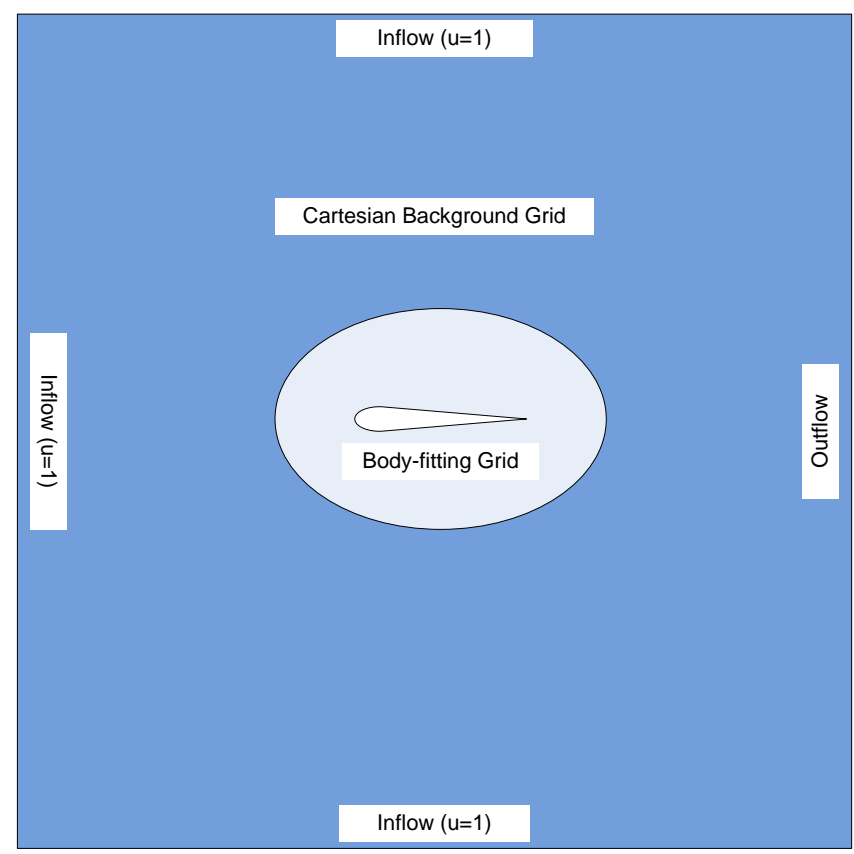

FIGURE 25 - Schematic of computational domain 
Once each airfoil was overlapped against the background grid, boundary conditions were applied to the walls of background grid. Uniform inflow boundary conditions were set on the top, bottom, and left walls with an inlet velocity of one. On the right wall, an outflow boundary condition was specified. The airfoil's surface was set as a no-slip wall. Figure 25 shows a simplified schematic of the computational domain.

At an angle of attack of $20^{\circ}$, the corrugated profile was tested with a $5 \mathrm{c} \times 5 \mathrm{c}$ and a $20 \mathrm{c} \times 20 \mathrm{c}$ domain. For the 20c x 20c grid, the grid density was increased to 640 x 640 to maintain the same grid cell size near the airfoil grid. Both setups were allowed to run long enough for a periodic flow pattern to develop Then the time averaged coefficient of lift and drag were then calculated from the force history of each using equations 6.1 and 6.2 respectively.

$$
\begin{aligned}
c_{L} & =\frac{L}{1 / 2 \rho U_{\infty}^{2} c} \\
c_{D} & =\frac{D}{1 / 2 \rho U_{\infty}^{2} c}
\end{aligned}
$$

Examining the coefficient of lift and drag time history in Figure 26 and Figure 27 respectively, it is possible to determine the effect of the domain size had on the airfoil's performance. It can easily be seen, the change in domain size had a very minor effect on the lift and drag histories of the corrugated profile. The 20x20 and the 5x5 line plots rest on top of one another showing that changing the domain size had a little to no effect on the unsteady flow around the profile.

Taking the time averaged coefficient of lift for both cases, it was found for the $5 \times 5$ was 0.948 and the $20 \times 20$ was 0.952 . The relatively small difference in coefficients of lift, show that setting the top and bottom boundaries to inflow boundaries did successfully negate any static blockage effects in the computational domain. Again, if the time averaged coefficient of drag for 
both were examined, it was found that the coefficient of drag was 0.439 and 0.445 respectively. Thus a 5c x 5c background grid was chosen.

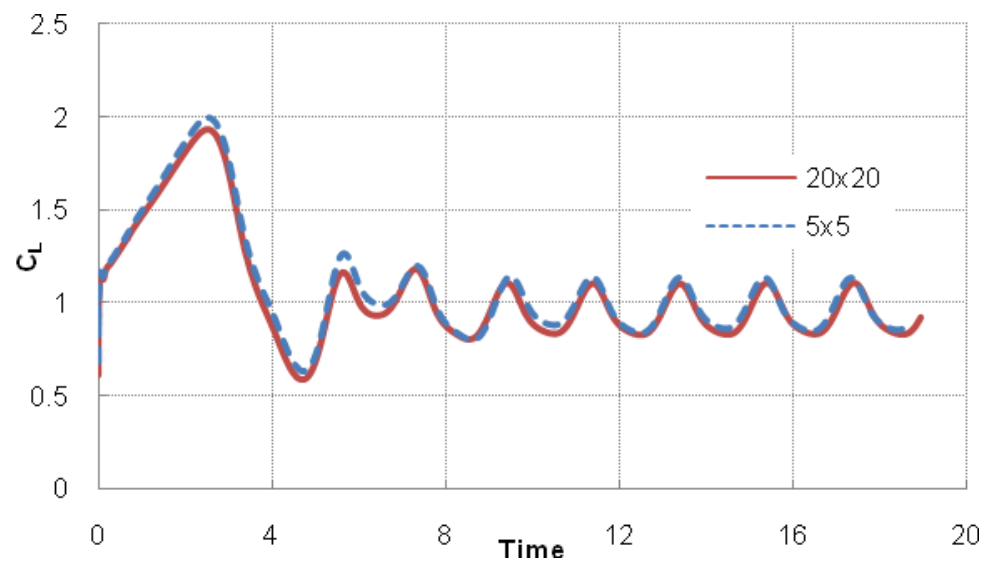

FIGURE 26 - Effect of domain size on coefficient of lift

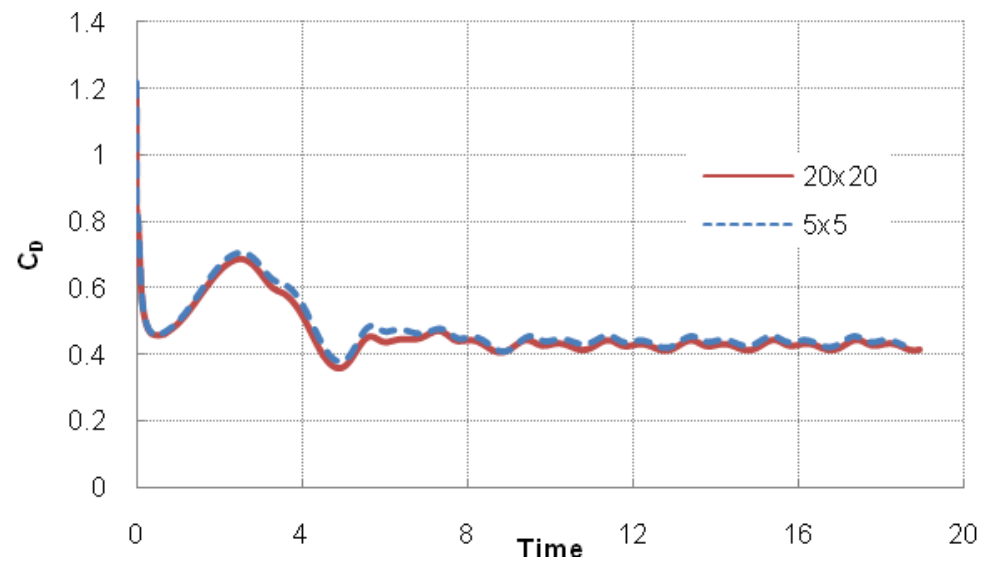

FIGURE 27 - Effect of domain size on coefficient of drag 


\section{Airfoil Grid Refinement}

The sensitivity analysis for the corrugated profile and the flat plate each followed the same methodologies. Each grid would have their grid densities varied in the circumferential and radial directions about the airfoil while holding an angle of attack of 10 degrees. Figure 28 illustrates the grid density direction definitions. Each grid variation was run long enough to allow either a steady state or periodic flow pattern to develop. The time averaged coefficient of lift and drag were then calculated from the force history of each trial using equations 6.1 and 6.2 respectively.

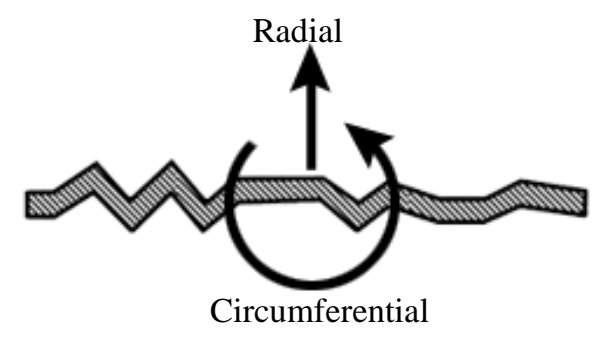

FIGURE 28 - Direction definition

The results are shown in Figure 29. It seemed that a grid with 600 lines in the circumferential direction and 200 lines in the radial (600x200) were required for the corrugate profile and a 400x100 grid for the flat plate. The corrugate profile requires a significantly higher density than the flat plate because of its more complex geometry. 

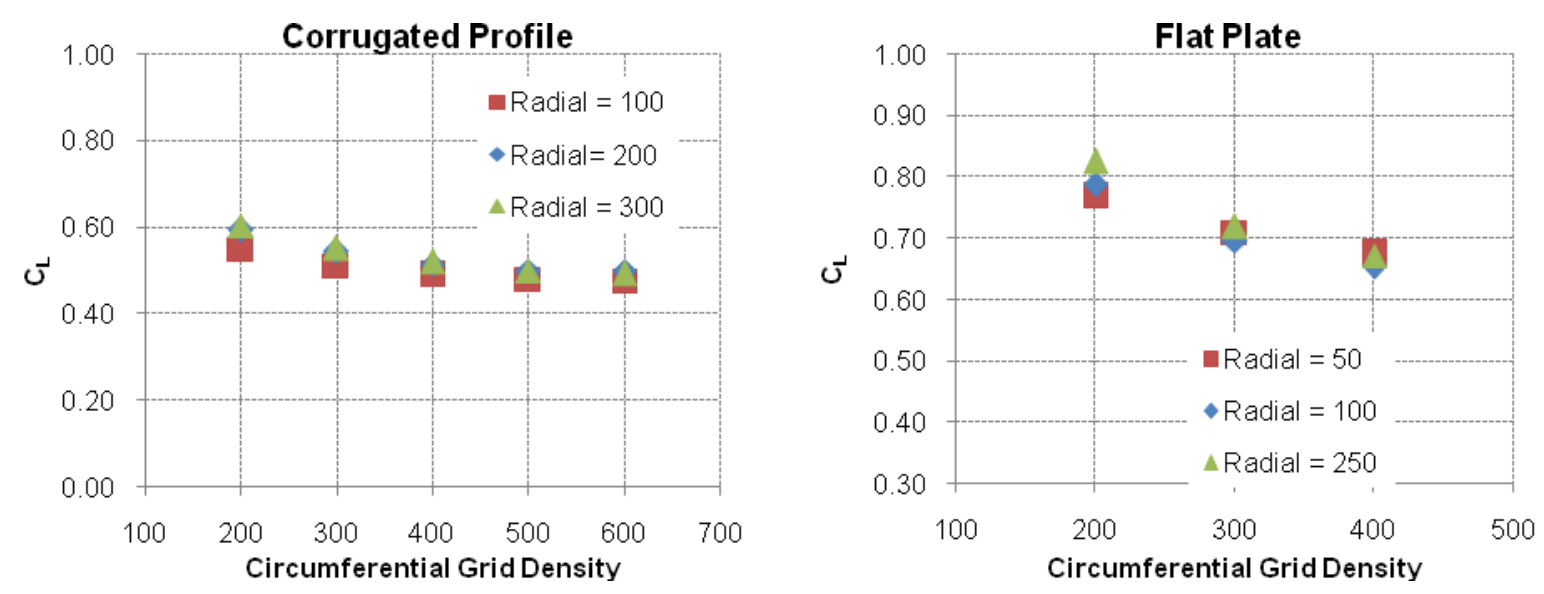

FIGURE 29 - The grid sensitivity analysis

However, by seeing convergence in the lift coefficient, it does not necessarily mean the grid system is sufficient. Upon examining the flow field around each airfoil, it was observed that velocity wake was quickly damped out as it passed into coarser grids in the wake region. In Figure 30(a) the dampening effect can easily seen by comparing the velocity contour and the grid together. As the cells of the grids become larger, the flow field is damped out at an increasing rate.

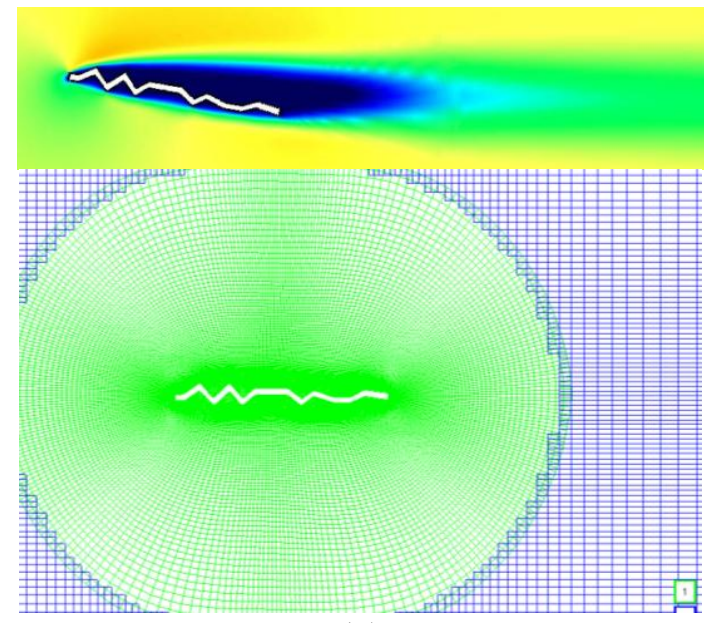

(a)

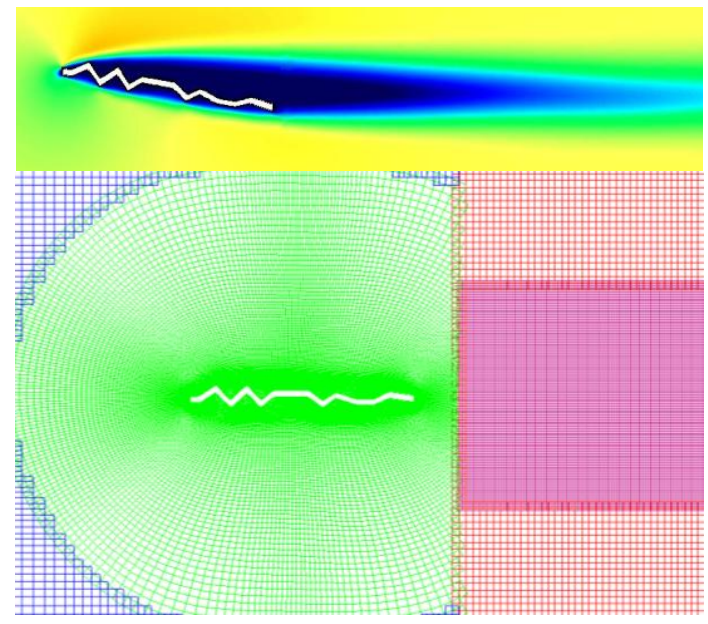

(b)

FIGURE 30- The U velocity contour

It was determined that an additional set of grids in the wake region would be needed to capture the velocity wake. The new set of grids consisted of two grids. One grid was made of a 
uniform set of cells approximately equal to the cell size $10 \%$ of the chord away from the airfoil, and the second grid assisted in interpolation of the first grid against the background grid. Figure 30(b) shows new grid (with added grid in the wake region) with respective streamwise velocity contours. It was clear that the added trailing edge grid could better resolve the flow structure in the wake. To fully understand the effect the new trailing grid had on the grid system, a second grid refinement study was conducted.

Instead of performing an in depth analysis that the radial density for the flat plate and the corrugated profile would remain at 100 and 200 respectively, and only the circumferential density of the airfoil would be varied. The primary trailing grid for corrugated profile and flat plate would be a 200x100 and 200x50 Cartesian grid respectively. The effects of the trailing grid analysis can be seen in Figure 31.

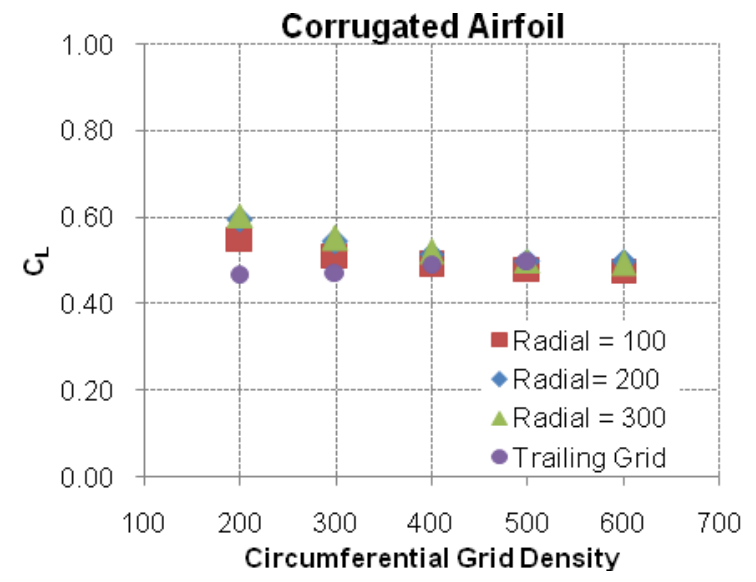

(a)

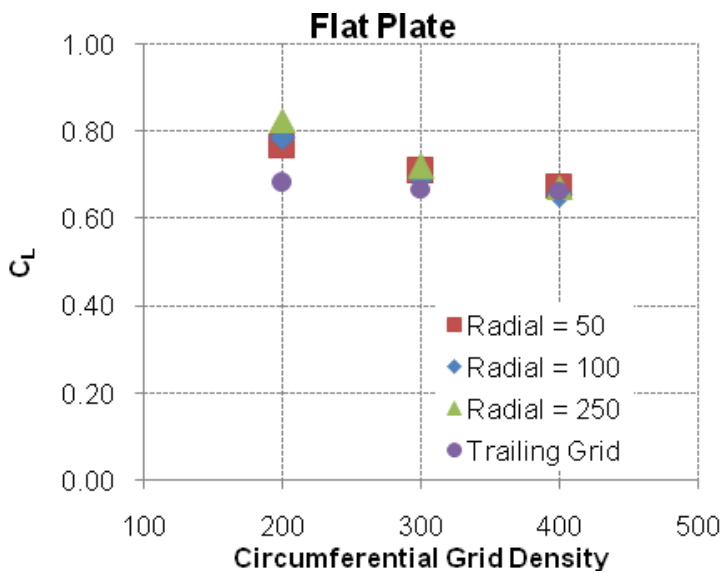

(b)

FIGURE 31 - The grid sensitivity with trailing grid

From each graph, it can be seen that the required grid density for each airfoil grid can be reduced with the trailing edge grid. From this analysis it was found a 400x100 corrugated profile grid with a 200x100 trailing edge grid would accurately recreate the necessary grid scheme to 
produce valid results, and preserve the flow field around the corrugate profile. Without the trailing edge grid, it would require a 600x200 airfoil grid. For the flat plate, it was found that a grid with a 300x100 airfoil grid with a 200x50 trailing grid was sufficient. While this reduction in grid points seems insignificant, it reduced the total number of nodes in the corrugated profile domain by $30 \%$ and the flat plate remained about equal. With the domain and each airfoil grid properly defined, it was then possible to conduct the initial aerodynamic study. 


\section{AERODYNAMIC ANALYSIS}

\section{A. Angle of Attack}

The effect of angle of attack on aerodynamic forces was investigated. The chord Reynolds number remained 1000 while the angle of attack was varied for $0^{\circ}$ to $40^{\circ}$. For comparison, the calculated coefficients of lift of the corrugated profile were plotted against experimental data from Kesel (1997) at Reynolds equal to 10,000 and numerical data from Kim et al. (2009) at Reynolds equal to 1400 in Figure 32(a) who both tested similar profiles. Since vortex shedding occurred at modest angles of attack, the results plotted are the time averaged coefficient of lift. The numerical results were very close to the experimental data at low angles of attack $\left(\alpha<=4^{\circ}\right)$. Because of the disparity of Reynolds number used, the closeness seems to indicate the lift was not sensitive to the Reynolds number at low angles of attack. At modest angles of attack there is a disagreement between Kim et al.'s and the numerical data. The simulation predicts a lower lift coefficient compared to Kim et al's measurement. The deviation from Kim et al's simulation may be explained by the corrugate profile they used. While their profile was identical, it had rounded leading and trailing edges. The effect this has on lift is unknown. One reason for the 
high lift in both the numerical results of the corrugated profile and Kim et al.'s data was that at low Reynolds number viscous played an important role and flow remained attached. No obvious stall was observed over the range studied.

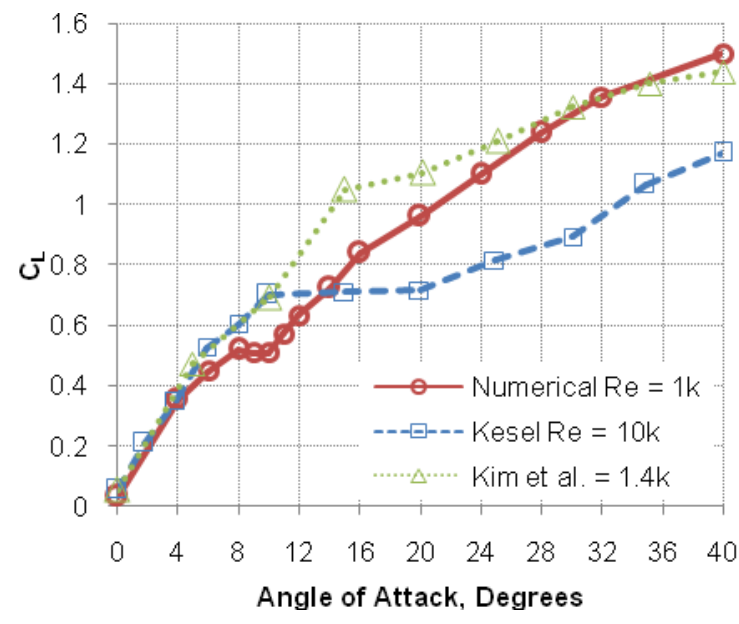

(a)

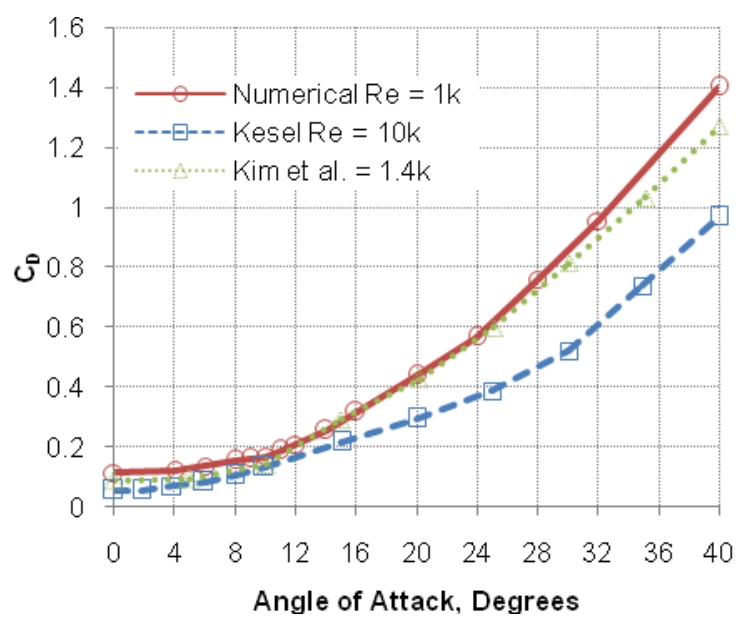

(b)

FIGURE 32 - Numerical results of corrugated profile plotted against published data.

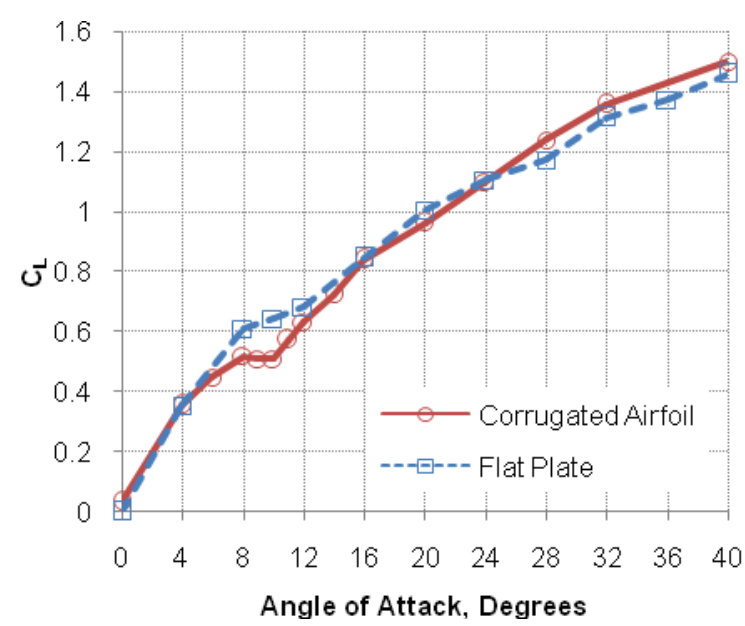

(a)

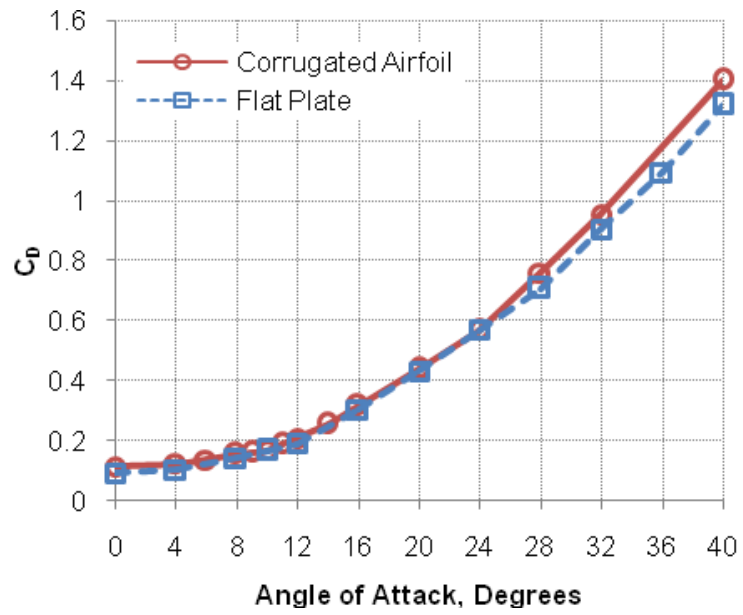

(b)

FIGURE 33 - Results plotted against numerical results of the flat plate.

Comparison with the flat plate is shown in Figure 33(c) and (d). The corrugated wing and the flat plate produce very similar lift at almost all the angles of attack, which was consistent with 
Kesel's finding which concluded that the corrugated profile acts much like a flat plate. The corrugated profile produced slightly higher drag than the flat plate. The slight deviation in lift between the corrugated profile and flat plate may be due to the difference in leading and trailing edges. The corrugated profile has sharp edges, while the flat plate has rounded. It is unknown how this affected the lift and drag across both profiles. 


\section{B. Effects of Corrugation}

Examining the streamlines around the corrugated airfoil, it was found that near stagnant rotating flow was trapped within the valleys. Due to trapped vortices, the corrugated airfoil acted like a thick and smooth airfoil. Figure 34 shows the virtual profile around the corrugation compared to the flat plate at various angles of attack. The increase in virtual thickness of the airfoil increased the pressure drag. The flat plate does not show a virtual profile thickness increase until approximately $8^{\circ}$. When the angle is less than $8^{\circ}$, the flow sticks to the surface of the flat plate, while the flow conveys across the vortices in the corrugated airfoil. This delay in virtual profile development reduces pressure drag, while the rotating flow in the valley results in the production of negative viscous drag that reduces the total drag caused by shear.

At $8^{\circ}$, the coefficient of drag for the corrugated airfoil and the flat plate were 0.156 and 0.141 respectively. The viscous drag made up only $17 \%$ of the total drag on the corrugated airfoil, while the viscous drag made up $30 \%$ of the drag on the flat plate. This effectively shows that the corrugated airfoil had lower viscous drag. However, because of the thick virtual profile produced by the trapped vortices, the pressure drag on the corrugated airfoil was 1.34 times higher than the flat plate's pressure drag. This increase in drag reduces the lift-to-drag ratio. Both the flat plate and the corrugated airfoil produced peak lift-to-drag ratios, $\left(\mathrm{c}_{\mathrm{L}} / \mathrm{c}_{\mathrm{D}}\right)_{\max }$, around $8^{\circ}$. The peak liftto-drag ratio of the flat plate and the corrugated airfoil were 4.3 and 3.3 respectively. 

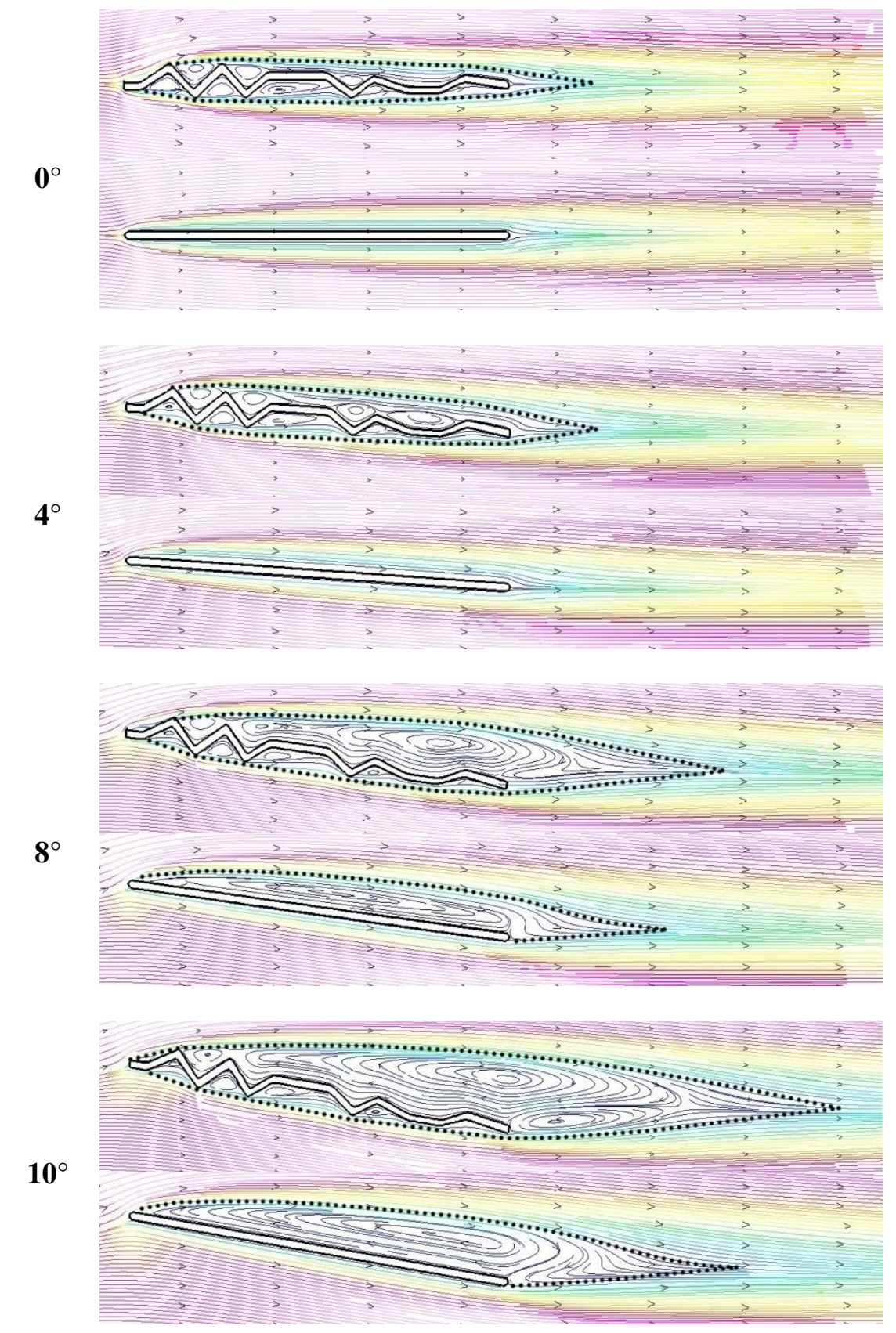

FIGURE 34 - Steady flow streamline visualization around the corrugated profile. Virtual profile represented by dotted line. 


\section{Stability of Corrugated Profile}

At angles greater than $12^{\circ}$, both the corrugated profile and the flat plate began to exhibit vortex shedding. The vortex shedding causes both lift and drag to oscillate on the airfoil. The Strouhal number of the vortex shedding at different angles of attack is plotted in Figure 35(a). Overall, the Strouhal number decreases with the angle of attack, which is consistent with the observation that the Strouhal number over a blunt body is approximately 0.2 . The corrugated wing and the flat plate demonstrate similar pattern at high angles of attack, indicating both of them behave like a blunt body. However at angles of $24^{\circ}$ and $28^{\circ}$ the leading edge vortex stays attached to the both the flat plate and corrugated profile, and only the trailing edge vortex sheds. This cuts the shedding frequency in half, thus reducing the Strouhal number by half. Figure 35(b) plots the maximum and minimum lift at each angle of attack for the corrugated profile and flat plate; the solid and hollow symbols representing the maximum and minimum coefficients of lift respectively.

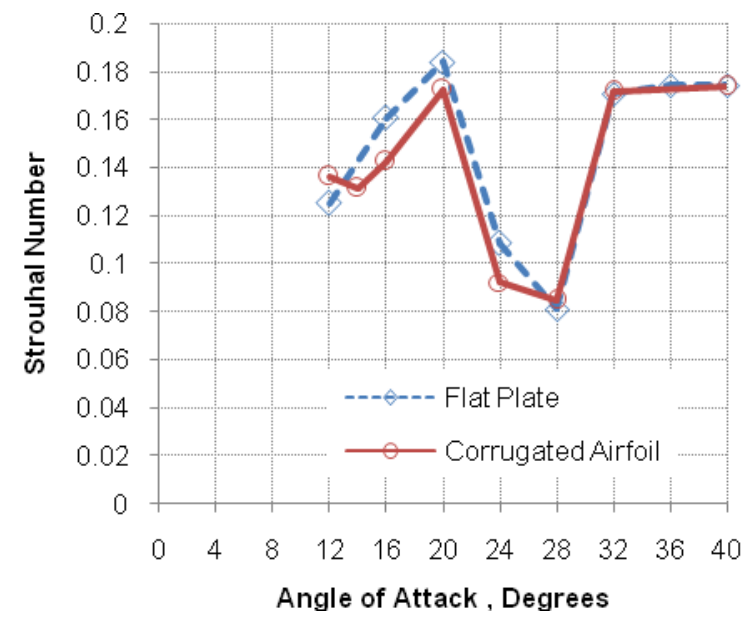

(a)

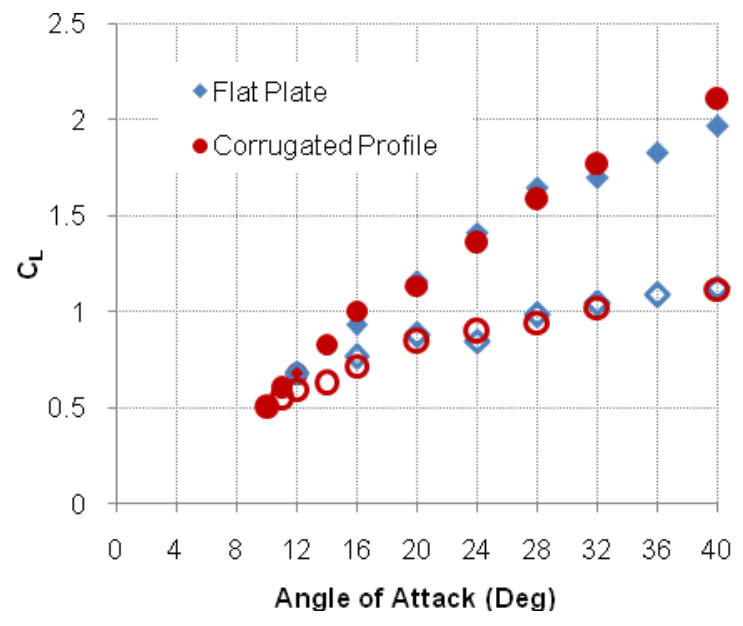

(b) 
FIGURE 35 - (a) Strouhal number of wake shedding frequency. (b) Oscillating coefficient of lifts

Comparing Figure 35(a) and Figure 35(b) it can be seen again how the corrugated profile acts very similar to the flat plate even in lift oscillation. The lift history plot in Figure 36 illustrates the similarities of the shedding frequency and lift oscillations in both the flat plate and corrugated airfoil at $20^{\circ}$. In Figure 37 are snapshots of the vorticity contours of both airfoils at $20^{\circ}$. The snapshots were taken at the time instants corresponding to maximum and minimum lift. It is important to note that the corrugation does not seem to affect the frequency.

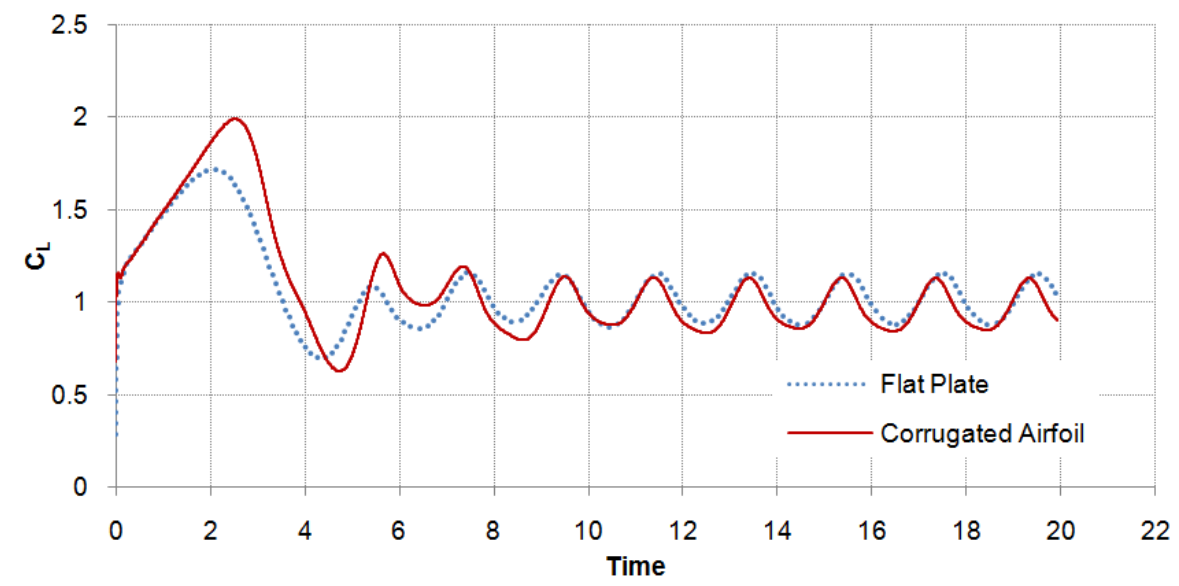

FIGURE 36 - Force history of Flat Plate and Corrugated Airfoil at $\mathbf{2 0}^{\circ}$
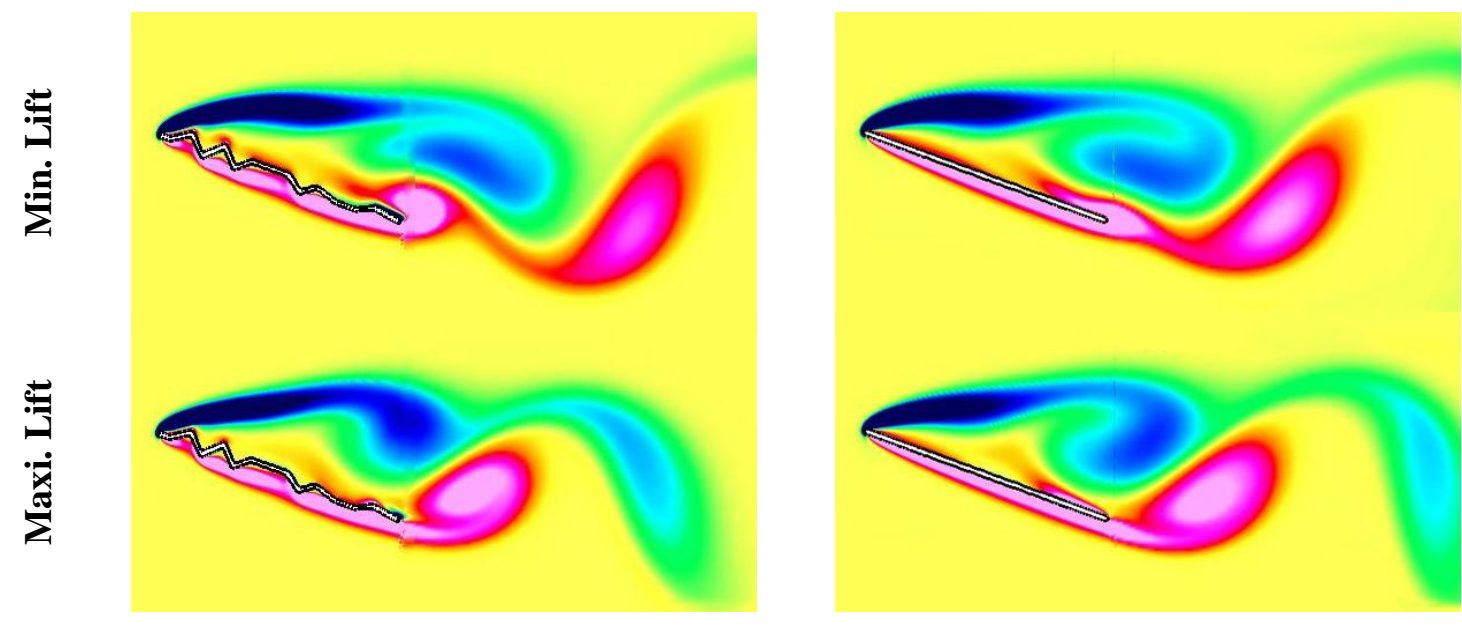

FIGURE 37 - Vorticity contours of the corrugated airfoil (left column) and flat plate (right column) at $2^{\circ}$ after periodic flow has been established. 


\section{Viability of Corrugated Profile}

The numerical simulations have demonstrated that the corrugated airfoil act similar in lift production as a flat plat in gliding conditions, however it produces more drag which agrees with Kesel (2000). This increase in drag is caused by the near stagnate rotating vortices in the valleys of the corrugation make the corrugated airfoil function like a thick and cambered streamlined airfoil. The airfoil thickness and camber increase the pressure drag. The increase in pressure drag is partially offset by the decrease in viscous drag due to the negative shear stresses in the valleys, leading to a net increase of total drag. This increase in drag reduces lift-to-drag ratio significantly. The corrugated profile has also exhibited similar vortex shedding frequencies and lift oscillation amplitudes as the flat plate. This evidence seems to indicate that the corrugated airfoil is not intended for aerodynamic improvement during gliding flight, but instead for structural benefits as previously hypothesized by other researchers. (Rees 1975, Rupolph 1977, Kesel 2000) 


\section{STRUCTURAL ANALYSIS}

Numerous studies have shown that insect wings are both a load bearing construction, and a flight mechanism. As an example, during the up and down stroke, the wing accelerates the surrounding fluid resulting in lift and thrust production, while the wing experiences a range of dynamic loading. Usually the wings do not exceed $1-2 \%$ of the total body mass of the insect. Therefore the wings must process the greatest possible load-bearing capacity possible, with the least amount of material. (Kesel 1997) The goal of this quick structural analysis is to show the advantages of using corrugation in a wing planform under static gliding flight.

\section{A. Material Properties and Modeling}

The structural simulation tests preformed were similar to Kesel et al.'s (1997) finite element analysis of the structural benefit of the Aeshna cyanea forewing. Instead of testing the forewing, it was decided to create a $3 \mathrm{D}$ homogeneous profile of the wing span to test the structural properties of the corrugated profile. The corrugated wing span was then compared to a flat plate wing span. Each wing was created using the SolidWorks 2009 designer, and then tested using the program's own structural simulation tools. 
To model a dragonfly's wing, the 3D wing was given the properties of Chitin. Each model was assumed to be made of a material with an isotropic nature. The properties of Chitin were taken from Kesel et al. (1997) which list the Young's modulus as $6.1 \mathrm{GN} / \mathrm{m}^{2}$ and an assumed Poisson's ratio of 0.25 . Each model was then subjected to a pressure load of $4.061 \times 10^{-6}$ $\mathrm{GN} / \mathrm{mm}^{2}$, which provides a homogenous wing loading that approximates the weight of the dragonfly.

Several models of the corrugation and flat plate were created, varying the percent thickness of each from $1 \%$ to $6 \%$. Each wing had a chord length of $1 \mathrm{~cm}$ and span of $4 \mathrm{~cm}$, which are approximations of the dimensions of the Aeshna cyanea forewing; Figure 38 shows a sample of the models produced.
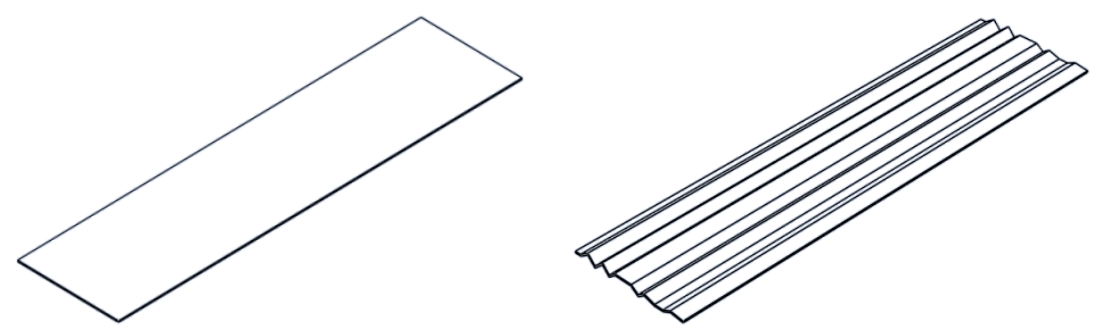

FIGURE 38 - The flat plate and corrugated wing models used. 


\section{B. Displacement and Stress}

The maximum displacement of the various profile thicknesses of the flat plate and corrugated wing were measured. In Figure 39(a) graphs the displacement of each thickness. The flat plate shows little resistance to displacement at its thinnest thickness. The $1 \%$ flat plate deflects approximately $2.5 \mathrm{~mm}$ under load. However, the corrugated profile demonstrates a much higher rigidity in comparison. Its deflection was 35 times lower than the flat plate. Table 1 details the deflection reduction between the corrugated profile and the flat plate at each thickness. Past a 3\% thickness, the flat plate shows little improvement on deflection reduction while the corrugated wing shows little to no change.

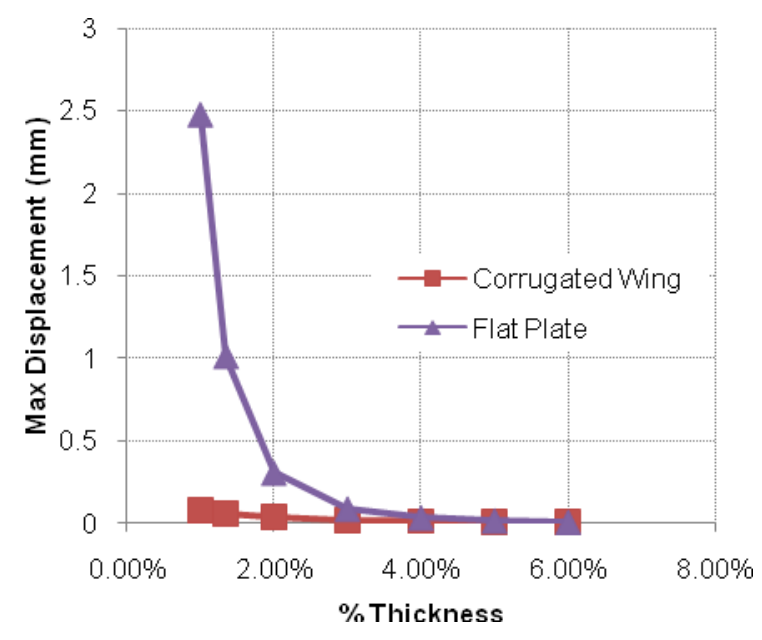

(a)

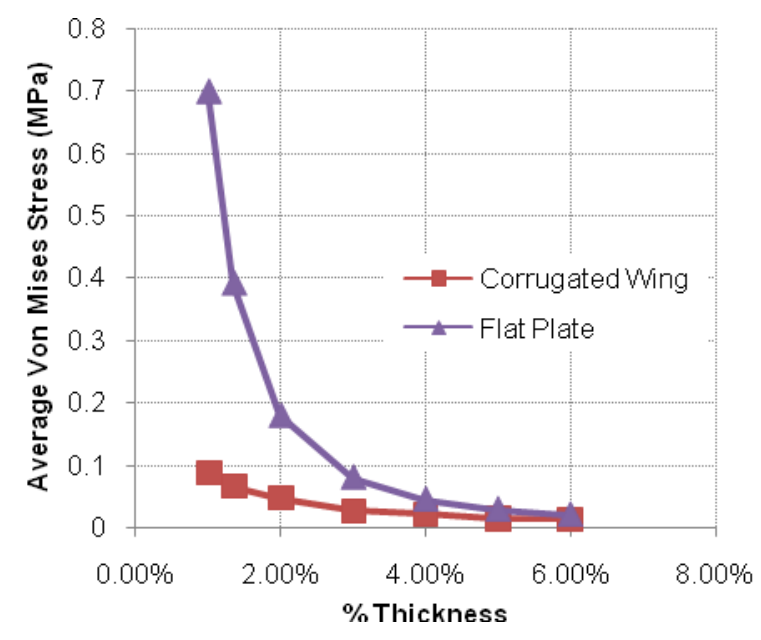

(b)

FIGURE 39 - (a) Max displacement of models (b) Average Von Mises Stress

A similar trend is seen with the average stress across the wings as previously seen with the deflection. The flat plate shows much higher average stress at lower thicknesses, but past $3 \%$ the reduction of stress decreases slower as the thickness increases. In Figure 39(b) graphs the average stress on each wing. At $1 \%$, the corrugated wing shows a reduction of stress by a factor 
of 8 . The corrugation again shows its superior ability to handle loading more efficiently than the flat plate without increasing the thickness of the wing. Like the deflection trend, the stress reduction ratio also begins to fall, as plate thickness increases. Table 1 shows the stress reduction at each thickness.

Table 2 details the equivalent percent thickness required for the flat plate to match the performance of the corrugated wing at each thickness ratio. It would take a $3.16 \%$ flat plate to match the displacement of a $1 \%$ corrugated wing.

\begin{tabular}{|c|c|c|c|c|}
\hline \multirow{2}{*}{$\begin{array}{c}\text { Corrugated } \\
\text { Wing } \\
\text { Thickness }\end{array}$} & \multicolumn{2}{|c|}{ Reduction Ratio } & \multicolumn{2}{c|}{$\begin{array}{c}\text { Equivalent Flat Plate } \\
\text { Thickness }\end{array}$} \\
\cline { 2 - 5 } & Displacement & Stress & Displacement & Stress \\
\hline $1.00 \%$ & 35.92 & 8.09 & $3.16 \%$ & $2.87 \%$ \\
$1.35 \%$ & 19.87 & 5.96 & $3.53 \%$ & $3.29 \%$ \\
$2.00 \%$ & 9.08 & 3.88 & $4.11 \%$ & $3.93 \%$ \\
$3.00 \%$ & 4.60 & 3.21 & $4.94 \%$ & $5.37 \%$ \\
$4.00 \%$ & 2.96 & 2.28 & $5.75 \%$ & $6.05 \%$ \\
$5.00 \%$ & 2.25 & 2.12 & $6.57 \%$ & $7.31 \%$ \\
$6.00 \%$ & 1.87 & 1.60 & $7.42 \%$ & $7.62 \%$ \\
\hline
\end{tabular}

Table 2. Equivalent thickness of flat plate to match performance of the corrugated wing. 


\section{CONCLUSION}

Numerical simulations were performed to understand the aerodynamic and structural characteristics of a corrugated airfoil. The aerodynamics was studied by solving the incompressible Navier-Stokes equations on an overlapping grid while the structure characteristics were investigated using commercial software SolidWorks 2009. It was observed that the corrugated profile produced similar lift as a flat plat, but at peak gliding ratios, it produces more drag. This increase in drag is due to the higher pressure drag resulting from the thicker virtual streamlined profile created by the stagnant vortices trapped in the valley. The higher pressure drag is partially offset by the negative shear stresses from the rotating vortices. The lift oscillations of both the flat plate and the corrugated profile were identical with similar vortex shedding frequencies at higher angles of attack. This shows that the corrugations do not interfere with the vortex shedding. However, compared to a flat plate, the flat plate produces more favorable aerodynamic characteristics. Overall, the aerodynamic analysis showed that the selected corrugated profile provides no advantages in terms of stall delay or lift generation in gliding flight. This conclusion is contrary to others' study at much higher Reynolds number. 
Investigating the structural properties of the corrugated profile and flat plate showed that under a static loading; the corrugated wing span had superior performance compared to the flat plate in terms of bending resistance. The study showed that the corrugated wing can reduce deflection and stress on the wing. It would take a flat plate with three times the thickness to perform as well as the corrugated wing. By using some degree of corrugation, it has been shown that increased structural rigidity can be obtained with minimum increase in materials.

These two studies provide strong evidence that the corrugated profile is primarily used for structural support in gliding flight at low Reynolds numbers. It provides similar lift, higher drag, and vastly more rigid lift surface. 


\section{References}

Aftosmis, M., Berger, M., and Melton, J., "Robust and efficient Cartesian mesh generation forcomponent-based geometry." AIAA Paper 97-0196-CP, 1997.

Benek, J., Buning ,P., and Steger, J., A 3d chimera grid embedding technique. AIAA Paper 85-1523-CP, 1985.

Brodsky, A. K. 1994. The Evolution of Insect Flight. New York: Oxford University Press.

Calhoun, D., and Wang, Z., "A Cartesian Grid Method for Solving the Two-Dimensional Streamfunction-Vorticity Equations in Irregular Regions," Journal of Computational Physics, Vol. 176, 2002, pp 231-275.

Chesshire, G. and Henshaw, W. "Composite overlapping meshes for the solution of partial differential equations.” Journal of Computational Physics, 90:1-64, 1990.

Choi, J., Oberoi, R., Edwards, J., and Rosati, J., "An Immersed Boundary Method for Complex Incompressible Flows," Journal of Computational Physiscs, Vol. 224, 2007, pp 757784.

Dou, H.S., Phan-Thien, N., "Parallelisation of an unstructured finite volume code with PVM: viscoelastic flow around a cylinder." Journal of Non-Newtonian Fluid Mechanics, Vol. 77, pp 21-51, 1998

Ellington C. P., "The aerodynamic of hovering insect flight: I. The quasi-steady analysis," Phil. Trans. R. Soc. Vol. 305, pp. 1-15

Gad-el-Hak, M. "Micro-Air-Vehicles: Can They Be Controlled Better?" Journal of Aircraft, Vol. 38, No. 3, May - June 2001, pp. 419-429.

Granlund, K., Ol, M., Garmann., D., Visbal, M., "Experiments and Computations on Abstractions of Perching," AIAA-2010-4943 
Guerrero, Joel. 2009. Numerical Simulation of the Unsteady Aerodynamics of Flapping Flight. Department of Civil, Environmental, Architectural Engineering, University of Genoa

Henshaw W., and Schwendeman D.,." An adaptive numerical scheme for high speed reactive flow on overlapping grids”. Journal of Computational Physics, 191:420-447, 2003.

Henshaw, W. "Ogen: An overlapping grid generator for overture." Technical report, Lawrence Livermore National Laboratory, Research Report UCRL-MA-132237, 1998.

Henshaw, W., "A Fourth-Order Accurate Method for the Incompressible Navier-Stokes Equations on Overlapping Grids," Journal of Computational Physics, Vol. 113, No. 1, pp. 13-25 1994

Henshaw, W., "On Multigrid for Overlapping Grid." SIAM Journal on Scientific Computing, 26:1547-1572, 2005.

Henshaw, W., Kreiss, H., and Reyna, L., "A Fourth-Order Accurate Method for the Incompressible Navier-Stokes Equations," Journal of Computers and Fluids, Vol. 23, 1994, 575593

HenshawW., "A solver for the incompressible navier-stokes equations. reference manual." Technical report, Lawrence Livermore National Laboratory, Research Report, 2006.

J. F. Thompson, B. K. Soni, and N. P. Weatherill. Handbook of Grid Generation. CRCPress, 1998.

Kesel, A., "Aerodynamic Characteristics Of Dragonfly Wing Sections Compared With Technical Aerofoils," The Journal of Experimental Biology, 2003, Vol. 200 PP. 543-556 1997

Kesel, A., Philippi, U., Nachtigall, W., "Biomechanical aspects of insect wing: an analysis using the finite element method," Computers in Biology and Medicine vol. 28, pp. 423-437, 1998

Kim, J., and Choi, H., "Distributed forcing of a flow over a circular cylinder,"Physics of Fluids, Vol. 17, Issue 3, 2005, pp. 033103-033103-16.

Kim, W., Ko, J., Park, H. Byun, D., "Effects of corrugation of the dragonfly wing on gliding performance," Journal of Theoretical Biology, Volume 260, Issue 4, 21 October 2009, pp.523530

McMasters, J. H., and Henderson, M. L., "Low Speed Single Element Airfoil Synthesis," Technical Soaring, Vol. 6, 1980, pp. 1- 21

Meakin, R., and Suhs, N., Unsteady aerodynamic simulation of multiple bodies in relative motion. AIAA Paper 89-1996-CP, 1989. 
Murphy, J., Hu, H., “An Experimental Investigation on a Bio-inspired Corrugated Airfoil," Experiments in Fluids, Vol. 49, pp 531-546, 2010

Noack, R., and Suggar, R., “A general capability for moving body overset cfd. In Proceedings of $7^{\text {th }}$ Symposium on Overset Composite Grid and Solution Technology, California, 2004.

Okamoto, M., Yasuda, K., and Azuma, A., "Aerodynamic Characteristics of the Wings and Body of a Dragonfly,” The Journal of Experimental Biology, Vol. 199, pp. 281-294, 1996

Posdziech, O., and Grundmann, R., "A systematic approach to the numerical calculation of fundamental quantities of the two-dimensional flow over a cylinder," Journal of Fluids and Structures, Vol. 23, 2007, pp 479-499.

Rees, C.J.C., "Aerodynamic Properties of An Insect Wing Section And A Smooth Aerofoil Compared," Nature, vol. 258 pp.141-142, 1975

Rudolph, R., "Aerodynamic Properties of Libella Quadrimaculata L. (Anisoptera. Libelludidea), And The Flow Around Smooth And Corrugated Wing Section Models During Gliding Flight," Odonatologica vol. 7, pp. 49-58 1977

Russel, D., and Wang, A.,"A Cartesian Grid method for Modeling Multiple Moving Objects in 2D Incompressible Flow,"Journal of Computational Physics, Vol. 191, 2003, pp 177-205.

SolidWorks 2009, x64 Edition SP3.0, Concord, Massachusetts, USA

Starius, G., "Composite mesh difference methods for elliptic boundary value problems." Numer. Math., 28:243-258, 1977.

Steger, J., Dougherty, F., and Benk, J., A chimera scheme; advances in grid generation. ASME FED, 5:59-69, 1983.

Sunada, S., Yasuda, T., Yasuda, K., Kawachi, K., "Comparison of Wing Characteristics at Ultralow Reynolds Number,” Journal of Aircraft, Vol. 39, No. 2, March-April 2002, pp. 331-338

Tannehill, J., Anderson, D., Pletcher, R., Computational Fluid Mechanics And Heat Transfer. Taylor \& Francis 1997

Tritton, D., "Experiments on the Flow Past a Circular Cylinder at Low Reynolds Numbers,"Journal of Fluids Mechanics, Vol. 6, 1959, pp 547-567.

Vargas, A. and Mittal, R. "A Computational Study of the Aerodynamic Performance of a Dragonfly Wing Section," AIAA 2004-2319, $2^{\text {nd }}$ Flow Control Conference, Portland, Oregon, 2004

Volkov, E., "A finite difference method for finite and infinite regions with piecewise smooth boundaries.” Doklady, 168:744-757, 1966. 
EDUCATION

AWARDS

EMPLOYMENT HISTORY

PUBLICATIONS

ACTIVITIES
M. Eng. in Mechanical Engineering

J.B. Speed School of Engineering, UofL, Louisville, KY

Thesis - Numerical Simulation of Corrugated Wing

Dates attended 8/2005 - present

B.S. in Mechanical Engineering

J.B. Speed School of Engineering, UofL, Louisville, KY

December 2009

Undergraduate GPA 3.12 / 4.0

\section{High School Diploma}

Franklin High School, Franklin, TN

May 2005

Speed School National Scholars Scholarship

Awarded to out of state students to assist with tuition

University of Louisville - Computational Fluid Dynamics Lab, Louisville, KY

Research Assistant

May 2009 - Present

- Perform analysis on corrugated wings at ultra-low Reynolds numbers

E.ON US -LG\&E -Project Engineering, Louisville, KY

Mechanical Co-op Engineer \#1

May - August 2008

- Design work on fuel delivery system for start up of power plant boiler.

- Coordinated hydro-excavation of cooling tower pipe for inspection.

E.ON US -LG\&E -Project Engineering, Louisville, KY

Mechanical Co-op Engineer \#2

August - December 2007

- Coordinated removal, and relocation of limestone processing equipment.

- Began preliminary design work for fuel delivery system for start up of super-critical power plant boiler.

- Spoke with Diesel fuel vendors of large delivery processes for fuel system

E.ON US -LG\&E -Project Engineering, Louisville, KY

Mechanical Co-op Engineer \#3

January - May 2007

- Assisting engineering's in locating and managing engineering drawings

- Began preliminary work of dust control for conversion to PRB-Coal conveyer systems.

Kyle Hord and Yongsheng Lian, "Numerical Investigation of the Aerodynamic and Structural Characteristics of a Corrugated Airfoil, " AIAA-2010-4624, 40th Fluid Dynamics Conference and Exhibit, Chicago, Illinois, June 28-1, 2010

\section{Cardinal Computational Fluid Dynamics}

Founder and President

April 2010-Present

- Registered Student Organization

- Arranged and organized student trips to conferences

- Managed laboratory personnel 